\title{
Recent Progress in Rare Oncogenic Drivers and Targeted Therapy For Non-Small Cell Lung Cancer
}

This article was published in the following Dove Press journal: OncoTargets and Therapy

\author{
Yijia Guo \\ Rui Cao \\ Xiangyan Zhang \\ Letian Huang \\ Li Sun \\ Jianzhu Zhao \\ Jietao Ma \\ Chengbo Han
}

Department of Oncology, Shengjing Hospital of China Medical University, China Medical University, Shenyang, People's Republic of China
Correspondence: Chengbo Han

Department of Oncology, Shengjing Hospital

of China Medical University, Shenyang

I I 0022, People's Republic of China

Tel +86 18940259860

Email hanchengbo@sj-hospital.org

\begin{abstract}
Non-small cell lung cancer (NSCLC) is frequently associated with oncogenic driver mutations, which play an important role in carcinogenesis and cancer progression. Targeting epidermal growth factor receptor (EGFR) mutations and anaplastic lymphoma kinase rearrangements has become standard therapy for patients with these aberrations because of the greater improvement of survival, tolerance, and quality-of-life compared to chemotherapy. Clinical trials for emerging therapies that target other less common driver genes are generating mixed results. Here, we review the literature on rare drivers in NSCLC with frequencies lower than 5\% (e.g., ROS1, RET, MET, BRAF, NTRK, HER2, NRG1, FGFR1, PIK3CA, DDR2, and EGFR exon 20 insertions). In summary, targeting rare oncogenic drivers in NSCLC has achieved some success. With the development of new inhibitors that target these rare drivers, the spectrum of targeted therapy has been expanded, although acquired resistance is still an unavoidable problem.
\end{abstract}

Keywords: non-small cell lung cancer, oncogenic driver, targeted therapy

\section{Introduction}

Lung cancer is the most common malignant disease with poor survival; the vast majority of cases are non-small cell lung cancer (NSCLC). The treatment strategy for NSCLC has been revolutionized by the discovery of molecular alterations that drive tumor initiation and progression. Epidermal growth factor receptor (EGFR), anaplastic lymphoma kinase (ALK), and KRAS are the most frequent oncogenic drivers of NSCLC, and the targeting of EGFR mutations and $A L K$ rearrangements has achieved great success. To date, the US Food and Drug Administration (FDA) has approved five EGFR tyrosine kinase inhibitors (TKIs) as the standard treatment for patients with activating EGFR mutations in NSCLC, including first-generation gefitinib and erlotinib, second-generation afatinib and dacomitinib, and thirdgeneration osimertinib. There are also currently five FDA-approved inhibitors of $A L K$ rearrangements, including first-generation crizotinib, second-generation ceritinib, alectinib, and brigatinib, and third-generation lorlatinib. The second- and third-generation inhibitors have exhibited enhanced activity against central nervous system (CNS) lesions and acquired resistance to crizotinib resulting from secondary ALK mutations. These achievements have led to clinical trials targeting less common driver genes, such as ROS1, RET, MET, BRAF, NTRK, HER2, NRG1, FGFR1, PIK3CA, DDR2, and EGFR exon 20 insertions (ins). In this review, we focus on these rare drivers and summarize their molecular biology, clinical features, targeted therapy, and acquired resistance. 


\section{ROSI Rearrangements}

Human proto-oncogene ROS1, which is located on chromosome band 6q22.1, is also known as MCF3 or c-ros-1. ${ }^{1,2}$ It encodes a receptor tyrosine kinase (RTK) that contains an extracellular or ectodomain, a single-pass transmembrane region with a hydrophobic stretch, and an intracellular carboxyl-terminal tyrosine kinase domain. ${ }^{3}$ Although the exact mechanisms by which ROS1 rearrangements promote carcinogenesis remain unclear, because most fusion partners of ROS1 lack dimerization domains, ${ }^{4} \mathrm{ROS} 1$ rearrangements are believed to promote signal transduction programs, proliferation, and cell survival through the upregulation of SHP-1 and SHP-2 and activation of the PI3K/AKT/mTOR, JAK/STAT, and MAPK/ERK pathways ${ }^{5-7}$ (Figure 1).

ROS1 fusion detection methods include fluorescence in situ hybridization (FISH), immunohistochemistry (IHC), reverse transcription-polymerase chain reaction (RT-PCR), and next-generation sequencing (NGS). FISH is the most common method, but formal screening recommendations for ROS1 fusions have not been established. ${ }^{4}$ ROS1 rearrangements are found in 1 to $2 \%$ of NSCLC. Over 14 types of ROS1 fusion partner genes have been reported, including CD74, SLC34A2, SDC4, EZR, FIG, TPM3, LRIG3, KDELR2, CCDC6, MSN, TMEM106B,
TPD52L, CLTC, and LIMA1, with the most frequent fusion partner being CD74 (40 to 45\%). ${ }^{4,8}$ Recent research found that patients with the CD74-ROS1 fusion were more susceptible to brain metastases and had lower objective response rates (ORR) to crizotinib than non-CD74-ROS1 patients, suggesting that the efficacy and prognosis of patients with advanced ROS1-rearranged NSCLC may be influenced by the type of ROS1 fusion partner. ${ }^{9}$ ROS1 fusions rarely overlap with other driver mutations, such as EGFR, ALK, or KRAS. ${ }^{10}$ ROS1 and ALK share a $49 \%$ amino acid sequence identity in the kinase domain and $77 \%$ identity in the adenosine triphosphate (ATP)-binding site, ${ }^{11}$ suggesting that ALK-TKIs may also inhibit ROS1. Similar to ALK, patients with ROS1-rearranged NSCLC are often younger, Asian ethnicity, have a non-smoking or light-smoking history, and advanced-stage disease with adenocarcinoma histology. ${ }^{12}$

\section{Crizotinib}

Crizotinib is a drug that targets ALK, ROS1, and MET, which has been used successfully to treat patients with advanced ALK-rearranged NSCLC. ${ }^{13,14}$ In a phase I study (PROFILE 1001) consisting of 50 patients with ROS1rearranged advanced NSCLC, the ORR for crizotinib was

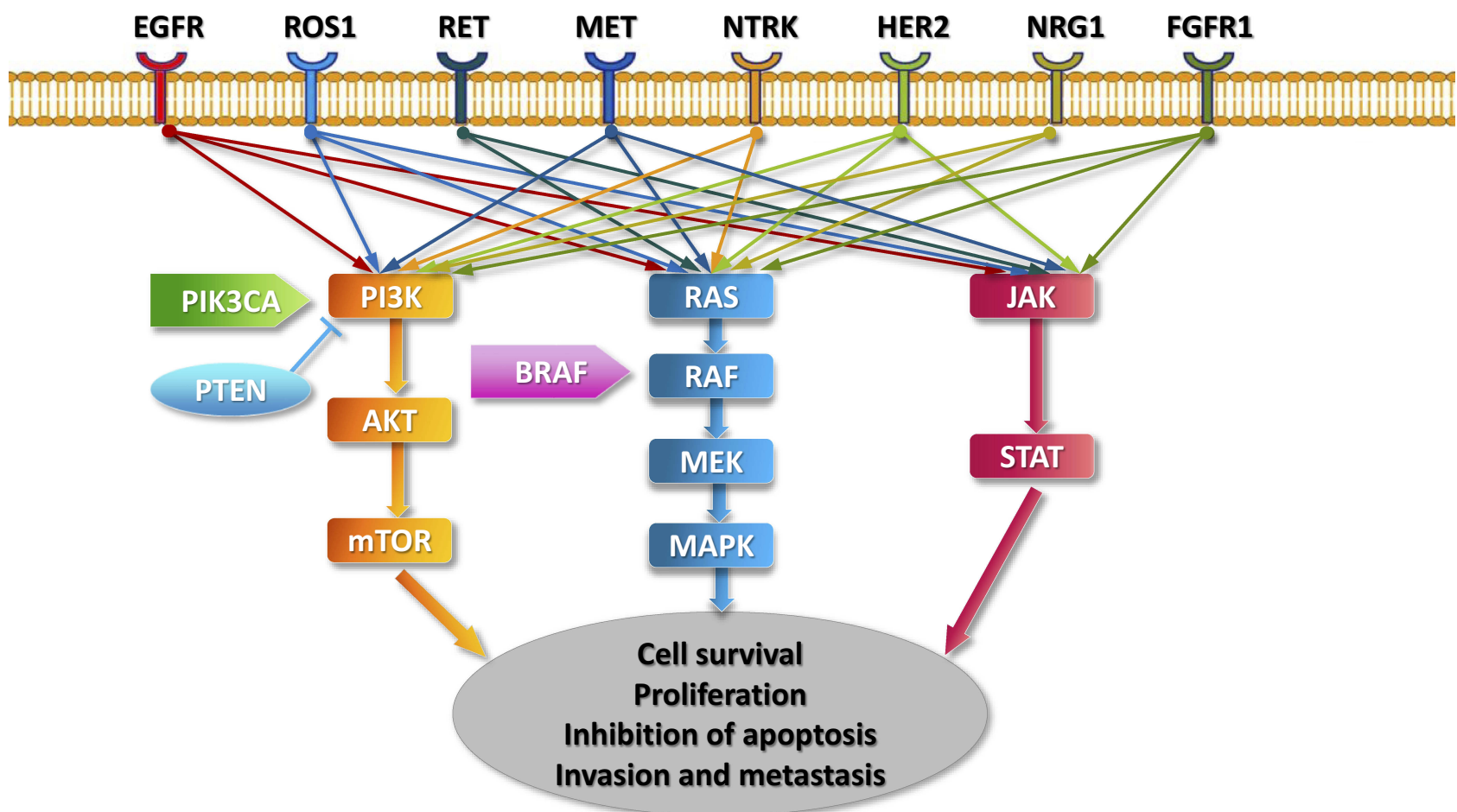

Figure I Key signaling pathways of oncogenic drivers in NSCLC. 
$72 \%$ with three complete responses and 33 partial responses, the median progression-free survival (PFS) was 19.2 months, and the safety profile of crizotinib was similar to that of patients with $A L K$-rearranged NSCLC ${ }^{11}$ (Table 1). Based on the study, crizotinib became the first targeted agent approved by the FDA for the treatment of advanced ROS1-rearranged NSCLC in March 2016. Crizotinib also demonstrated high response rates $(71 \%$ to $80 \%$; median PFS 9 to 10 months) in ROS1-rearranged NSCLC in two additional studies. ${ }^{15,16}$ Since 2017, National Comprehensive Cancer Network (NCCN) guidelines recommend that crizotinib be used for patients with known ROS1 rearrangements (grade $2 \mathrm{~A}$ recommendation). ${ }^{17}$

Similar to ALK or EGFR inhibition, acquired resistance is an important issue for the inhibition of ROS1. Mechanisms of acquired crizotinib resistance include acquired secondary mutations in the ROS1 kinase domain, bypassing signaling activation, and phenotypic changes. Mutations within the ROS1 kinase domain occur in 50 to $60 \%$ of crizotinibresistant tumors. ${ }^{18}$ The ROS1 G2032R mutation, which is analogous to ALK G1202R, has been the most frequent mutation identified. ${ }^{18-20}$ Additional mutations reported in clinical samples include D2033N (analogous to ALK $\mathrm{D} 1203 \mathrm{~N}$ ), S1986Y/F (analogous to ALK C1156Y), ${ }^{18,21}$ L2026M (analogous to ALK L1196M), and L1951R (no known analogous mutation in ALK). ${ }^{22}$

\section{Ceritinib}

Ceritinib is a potent and selective ALK inhibitor that also inhibits ROS1. In a phase II study (NCT01964157), 32 patients with ROS1-rearranged advanced NSCLC were treated with ceritinib, the ORR was $62 \%$ and disease control rate (DCR) was $81 \%$. The median PFS in this study was 9.3 months for all patients and 19.3 months for crizotinib-naïve patients with a median OS of 24 months. In eight patients with brain metastases, the intracranial ORR was $25 \%$ with an intracranial DCR of $63 \%{ }^{23}$ Based on the efficacy and safety demonstrated in this study, ceritinib became the second targeted agent recommended by the NCCN Guidelines for the treatment of advanced ROS1-rearranged NSCLC since November 2017. However, the toxicities of ceritinib should be taken into consideration due to the higher frequency of adverse events, such as diarrhea, nausea, anorexia, and vomiting compared to crizotinib. ${ }^{10}$

\section{Entrectinib (RXDX-I0I)}

Entrectinib is a multikinase inhibitor that targets ROS1, ALK, and tropomyosin receptor kinase (TRK) rearrangements and can effectively penetrate the blood-brain barrier. ${ }^{24,25}$ An integrated analysis of three studies (the phase II STARTRK-2 trial, phase I STARTRK-1 study, and the phase I ALKA-372-001 trial) for entrectinib in ROS1-positive NSCLC was presented at the 2018 World Conference on Lung Cancer (WCLC). For this analysis, 53 ROS1-rearranged and ROS1-inhibitor-naive NSCLC patients were evaluated, and the ORR was $77.4 \%$, the intracranial ORR was $73.9 \%$, the median duration of response (DOR) was 24.6 months, and the median PFS was 19.0 months (without CNS metastases: 26.3 months; with CNS metastases: 13.6 months). ${ }^{26}$ Early research found that entrectinib did not show activity against the ROS1 resistance mutations L2026M, G2032R, and D2033N. ${ }^{24,25}$ Based on these results, entrectinib has recently been recommended by the NCCN Guidelines for the first-line treatment of advanced ROS1-rearranged NSCLC. ${ }^{27}$ The global phase II STARTRK2 trial (NCT02568267) is still ongoing.

\section{Lorlatinib}

Second-line TKIs are being developed, such as lorlatinib, which was developed to target crizotinib-resistant $A L K$ rearranged NSCLC. It has in vitro activity against several crizotinib-resistant mutations, including $\mathrm{L}_{2026 \mathrm{M}}{ }^{28,29}$ $\mathrm{S} 1986 \mathrm{Y} / \mathrm{F}^{28}$ and $\mathrm{D} 2033 \mathrm{~N} .^{21}$ In a phase I trial for $A L K$ and ROS1-rearranged NSCLC, the 12 patients with ROS1rearranged lung adenocarcinomas had an ORR of $50 \%$ with a median PFS of seven months. ${ }^{30}$ In a phase II trial of ROS1-positive patients, $70 \%$ of which were crizotinibresistant, lorlatinib produced an overall ORR of $36.2 \%$, intracranial ORR of $56 \%$, and a median PFS of 9.6 months. ${ }^{31}$ Thus, lorlatinib has been recommended by the NCCN Guidelines for the treatment of advanced ROS1rearranged NSCLC that progressed after crizotinib, entrectinib or ceritinib. ${ }^{27}$

\section{DS-605 Ib}

DS-6051b is an oral, small molecule TKI with high affinity for ROS1 and NTRK kinases. In a phase I study (NCT02675491) evaluating DS-6051b in 15 Japanese patients with ROS1-rearranged NSCLCs, the ORR was $58.3 \%$ in patients with assessable target lesions and $66.7 \%$ in crizotinib-naïve patients, and the DCR was $100 \%{ }^{32}$ Another phase I/Ib study (NCT02279433) of DS-6051b in advanced solid tumors is ongoing.

\section{Repotrectinib (TPX-0005)}

Repotrectinib (TPX-0005) is a potent ALK/ROS1/TRK inhibitor that demonstrated encouraging clinical activity 


\begin{tabular}{|c|c|c|c|c|c|c|c|c|c|c|c|c|c|}
\hline 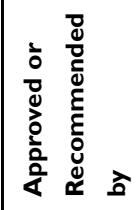 & 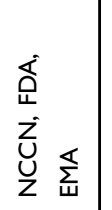 & $\begin{array}{l}Z \\
\text { U }\end{array}$ & 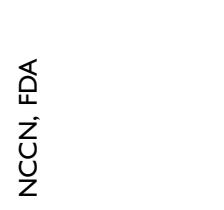 & $\begin{array}{l}Z \\
U \\
Z\end{array}$ & & & & $\begin{array}{l}\text { 己̌ } \\
\text { Z }\end{array}$ & $\begin{array}{l}\mathcal{Z}_{U}^{*} \\
\mathrm{U}\end{array}$ & $\begin{array}{l}\text { Zu }_{U}^{*} \\
\text { Z }\end{array}$ & & & 迆 \\
\hline $\begin{array}{l}\frac{\underline{y}}{\vec{z}} \\
\stackrel{\vec{y}}{x} \\
\ddot{x}\end{array}$ & 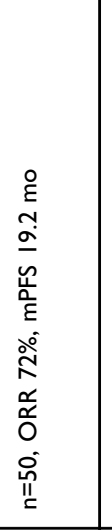 & 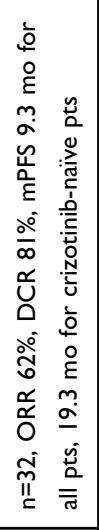 & 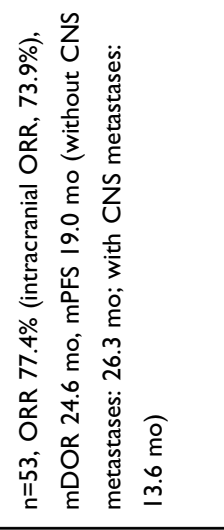 & 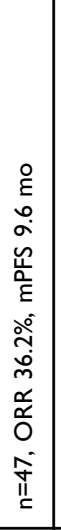 & 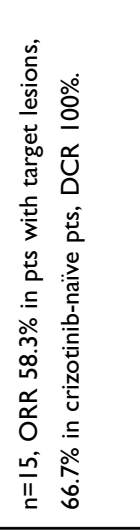 & $\begin{array}{l}\text { :0 } \\
\text { :0 } \\
\text { a. } \\
0 \\
\end{array}$ & 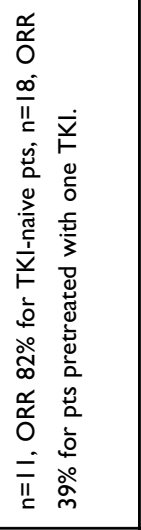 & 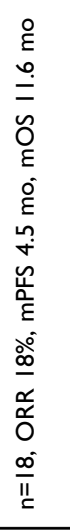 & 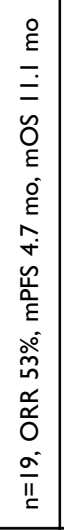 & 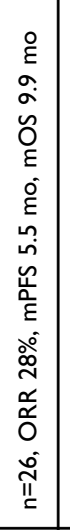 & 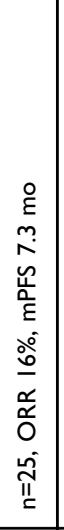 & 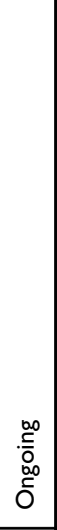 & 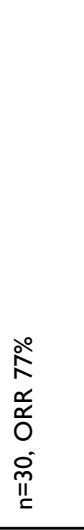 \\
\hline 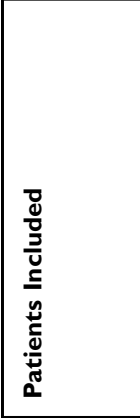 & 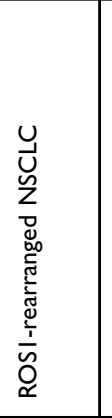 & 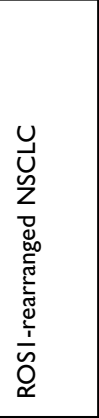 & 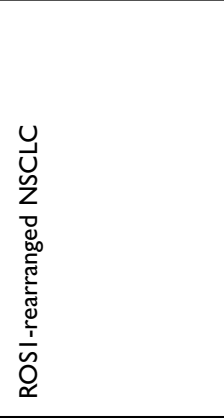 & 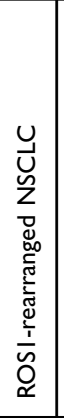 & 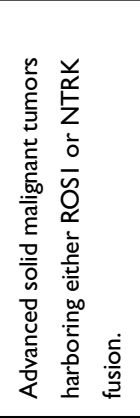 & 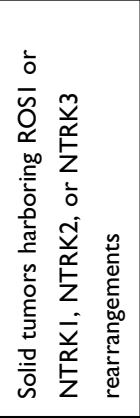 & 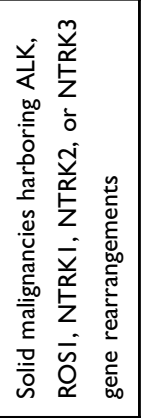 & 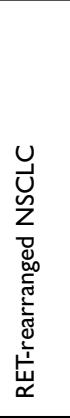 & 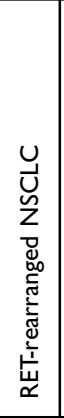 & 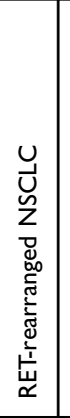 & 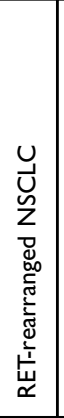 & 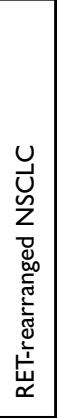 & 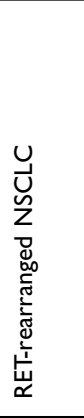 \\
\hline$\frac{\dddot{g}}{\frac{\pi}{\alpha}}$ & - & $=$ & $\equiv$ & $=$ & - & $\stackrel{\varrho}{\equiv}$ & $\equiv$ & $=$ & $=$ & $=$ & $=$ & $\equiv$ & $\equiv$ \\
\hline 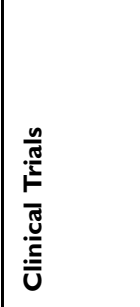 & 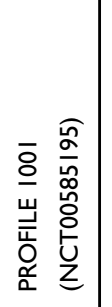 & 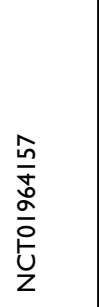 & 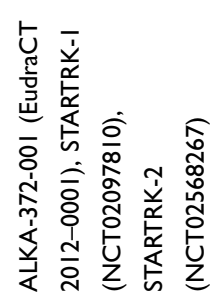 & 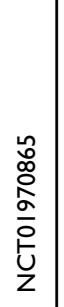 & 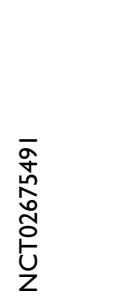 & 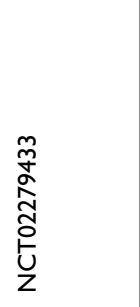 & 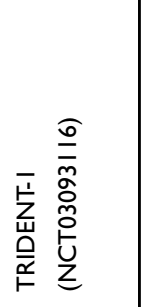 & 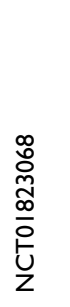 & 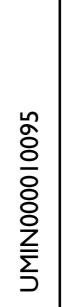 & 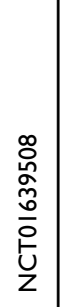 & 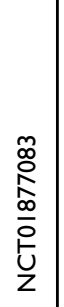 & $\begin{array}{l}\frac{\mathscr{Q}}{\frac{1}{m}} \\
\frac{m}{\tilde{m}} \\
\frac{\underline{Q}}{z}\end{array}$ & 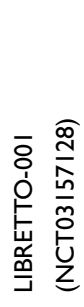 \\
\hline 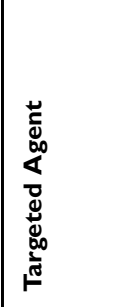 & 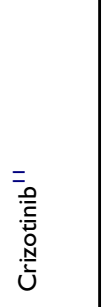 & Uू & 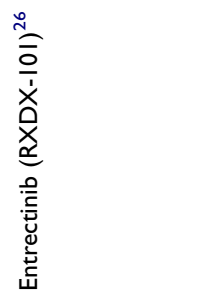 & 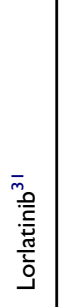 & 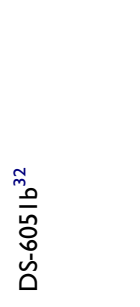 & 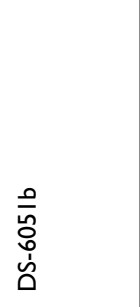 & 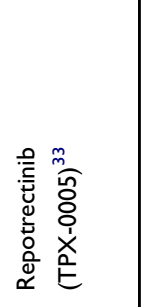 & 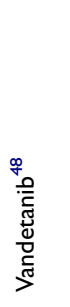 & 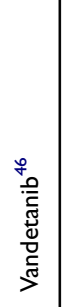 & 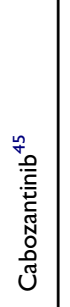 & 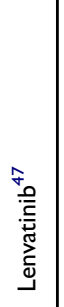 & 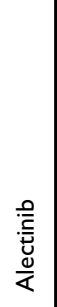 & 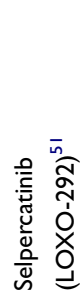 \\
\hline 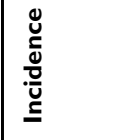 & \ે & & & & & & & \ે & & & & & \\
\hline 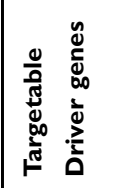 & 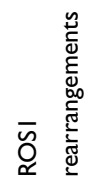 & & & & & & & $\underset{\check{\Sigma}}{\breve{w}}$ & & & & & \\
\hline
\end{tabular}




\begin{tabular}{|c|c|c|c|c|c|c|c|c|c|c|c|}
\hline \multicolumn{2}{|l|}{ 造 } & 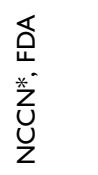 & 顿 & \multicolumn{2}{|l|}{ 顿 } & & & & & & \\
\hline 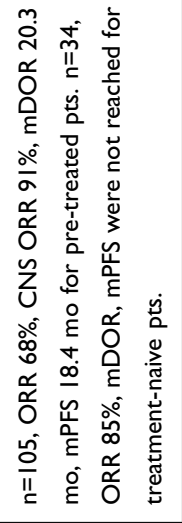 & 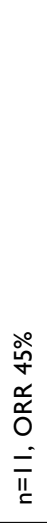 & 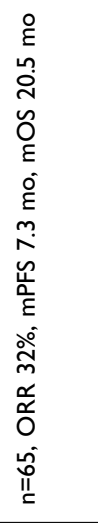 & 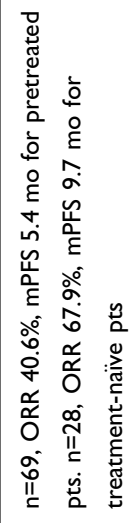 & 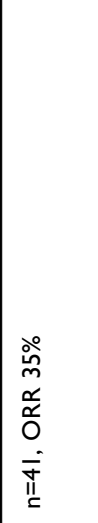 & 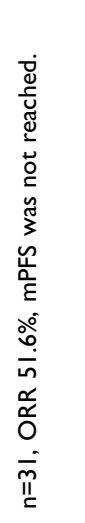 & 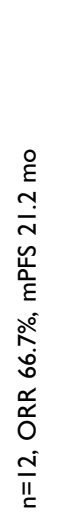 & 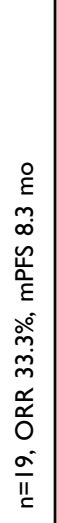 & 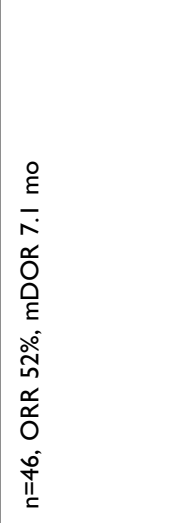 & 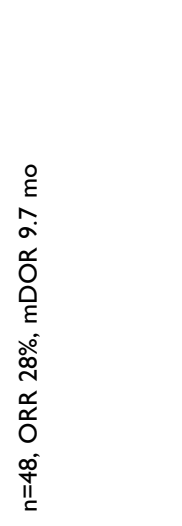 & 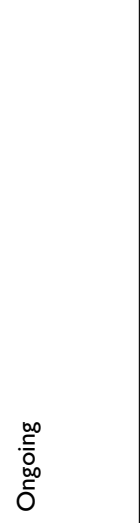 & 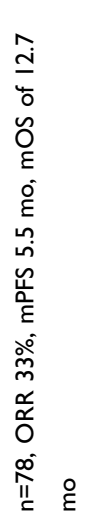 \\
\hline 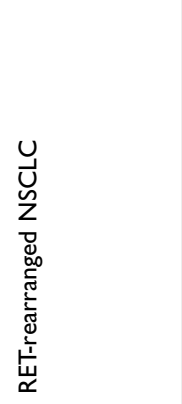 & 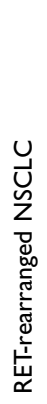 & 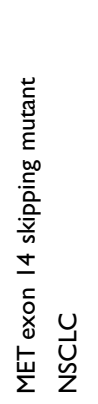 & 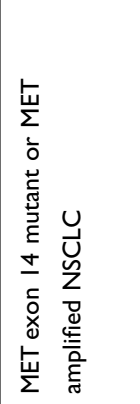 & 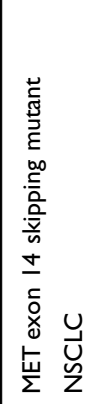 & 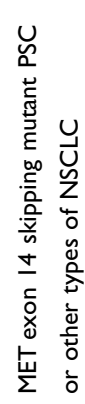 & 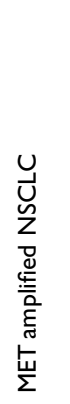 & 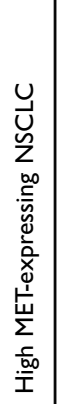 & 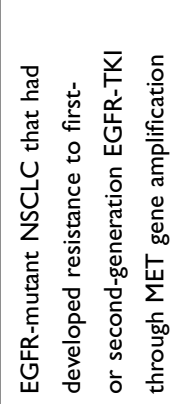 & 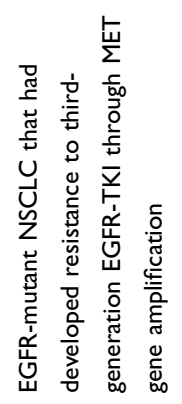 & 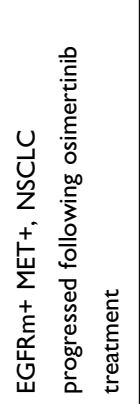 & 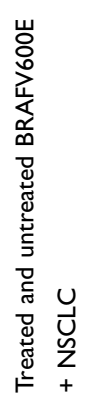 \\
\hline$\equiv$ & - & - & $=$ & $=$ & $=$ & $\underline{\overline{\underline{\underline{S}}}}$ & $\underline{\overline{\underline{\underline{I}}}}$ & $\stackrel{\rho}{ }$ & $\stackrel{\rho}{ }$ & $=$ & $=$ \\
\hline 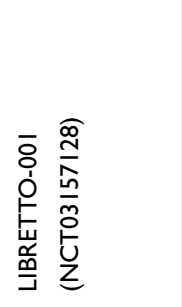 & 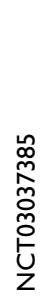 & 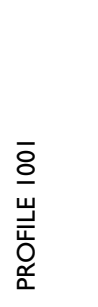 & 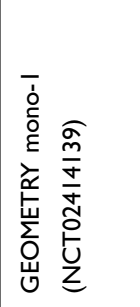 & 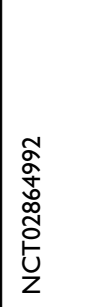 & 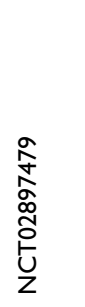 & 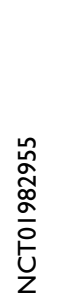 & 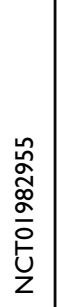 & 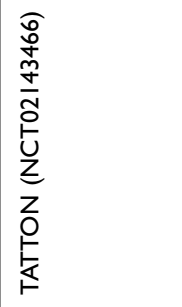 & 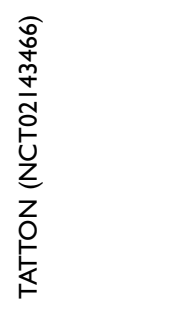 & 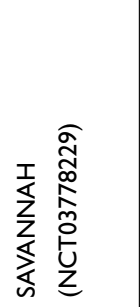 & 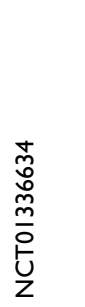 \\
\hline 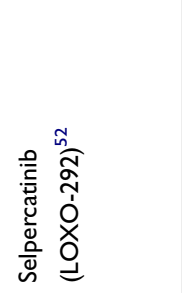 & 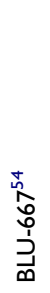 & 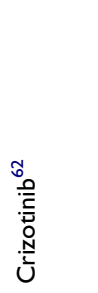 & 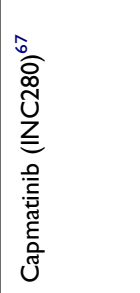 & 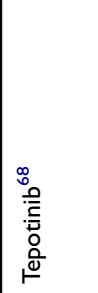 & 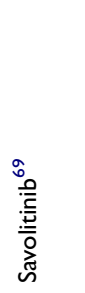 & 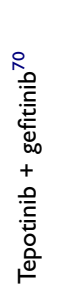 & 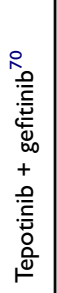 & 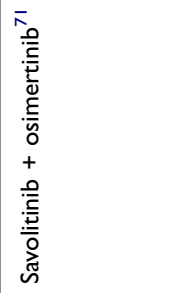 & 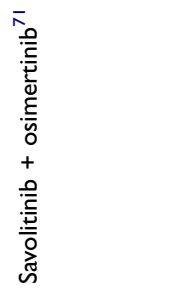 & 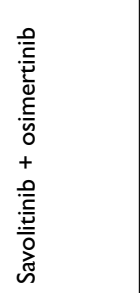 & 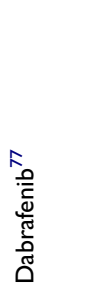 \\
\hline & & ஓ̊ & & & & $\stackrel{\circ}{\stackrel{\circ}{1}}$ & & & & & 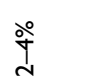 \\
\hline & & 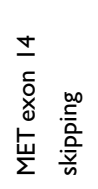 & & & & 崖 & & & & & 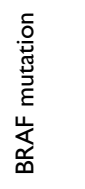 \\
\hline
\end{tabular}




\begin{tabular}{|c|c|c|c|c|c|c|c|c|c|c|c|c|}
\hline 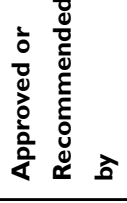 & 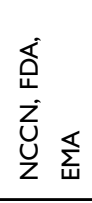 & 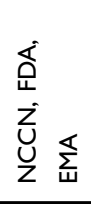 & 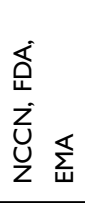 & & 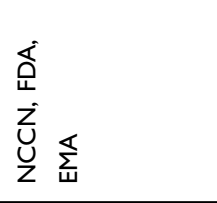 & & & & & $\begin{array}{l}\text { 艺 } \\
\text { Z }\end{array}$ & & \\
\hline$\frac{y}{\underline{x}}$ & 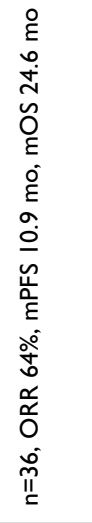 & 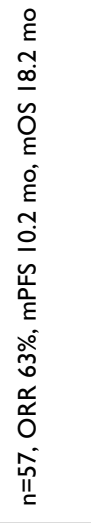 & 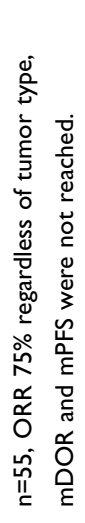 & 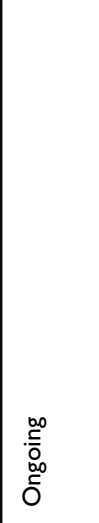 & 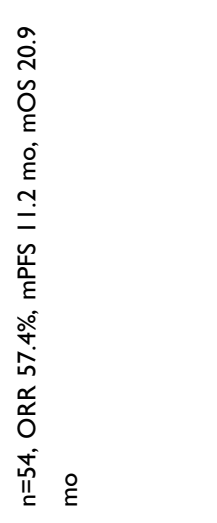 & 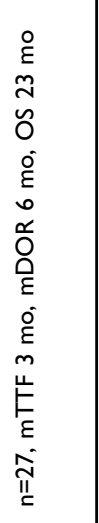 & 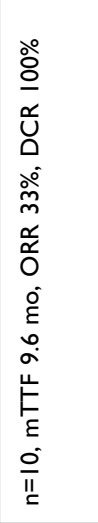 & 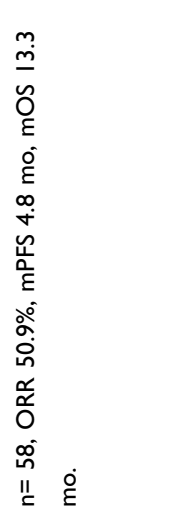 & 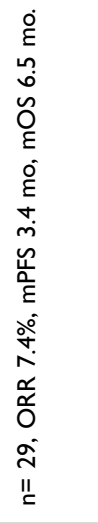 & 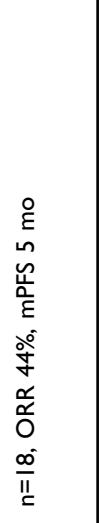 & 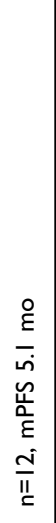 & 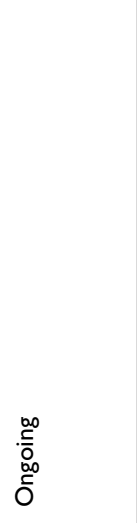 \\
\hline 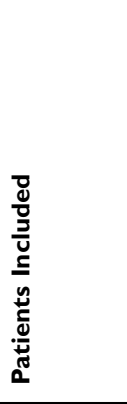 & 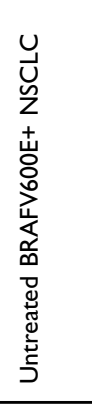 & 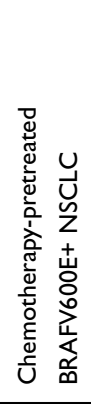 & 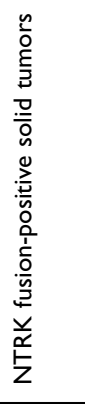 & 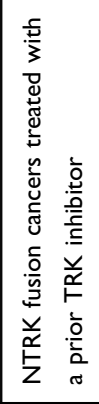 & 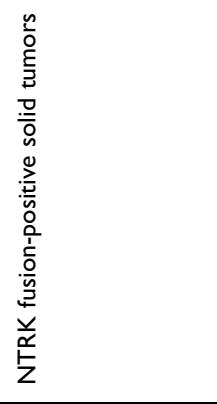 & 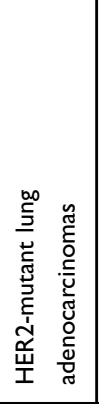 & 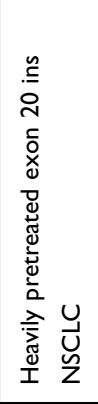 & 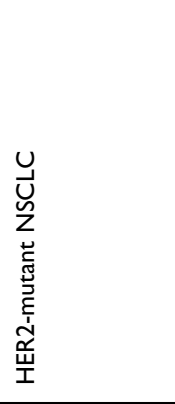 & 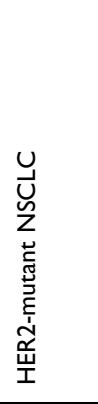 & 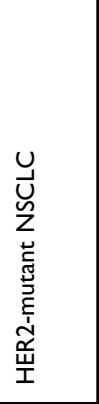 & 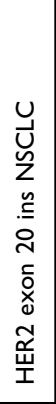 & 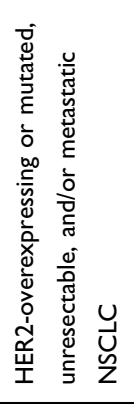 \\
\hline $\begin{array}{l}\frac{\Xi}{0} \\
\frac{c}{2}\end{array}$ & $=$ & $=$ & $=$ & $\equiv$ & $\equiv$ & \multirow[b]{2}{*}{ 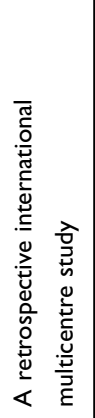 } & \multirow{2}{*}{ 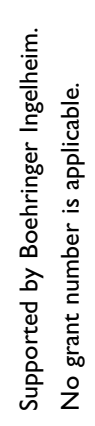 } & \multicolumn{3}{|r|}{$=$} & $=$ & $=$ \\
\hline 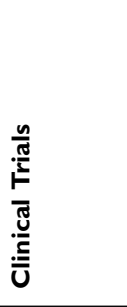 & 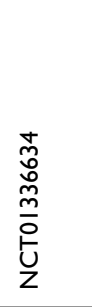 & 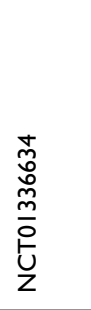 & 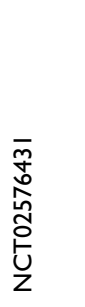 & $\begin{array}{l}\overline{\bar{n}} \\
\frac{\hat{n}}{\tilde{N}} \\
\hat{\tilde{\rho}} \\
\underline{U} \\
z\end{array}$ & 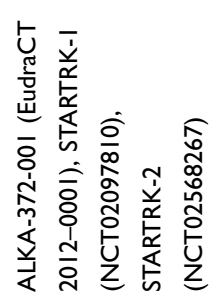 & & & 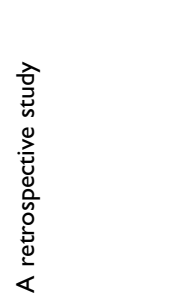 & 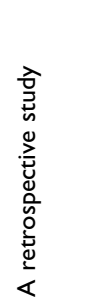 & 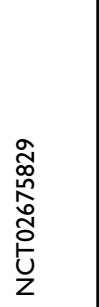 & 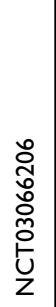 & 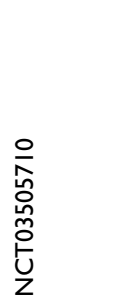 \\
\hline 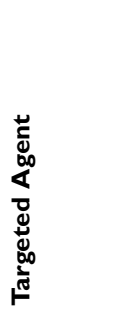 & 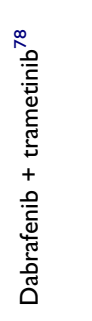 & 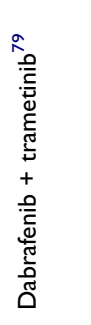 & 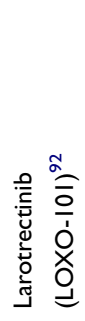 & 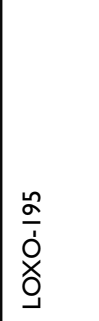 & 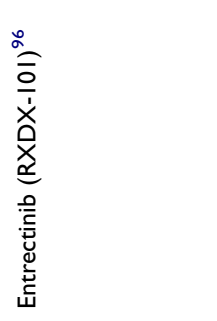 & 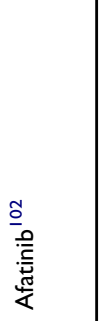 & 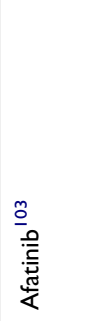 & 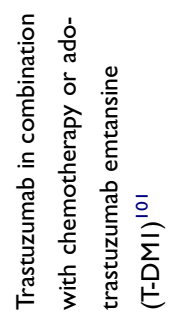 & 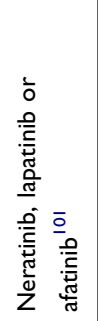 & 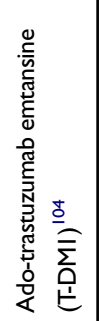 & 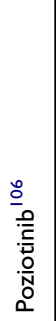 & 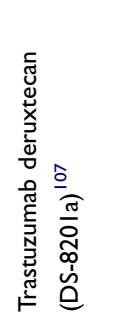 \\
\hline 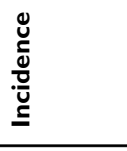 & & & ఫे & & & $\stackrel{\text { ఫे }}{\text { ปे }}$ & & & & & & \\
\hline 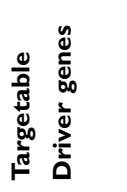 & & & 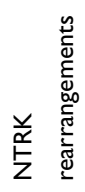 & & & 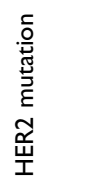 & & & & & & \\
\hline
\end{tabular}




\begin{tabular}{|c|c|c|c|c|c|c|c|c|c|c|c|}
\hline 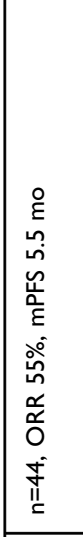 & 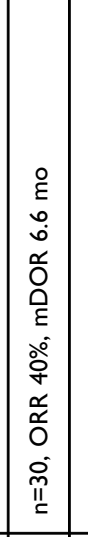 & 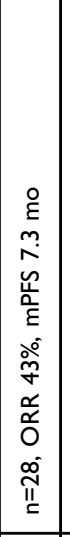 & 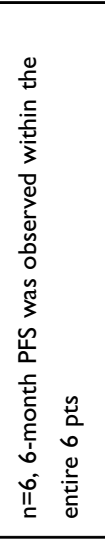 & 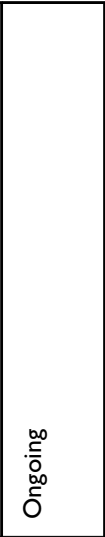 & 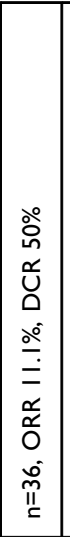 & 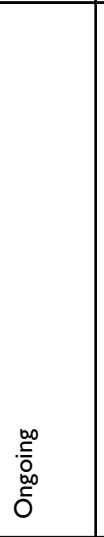 & 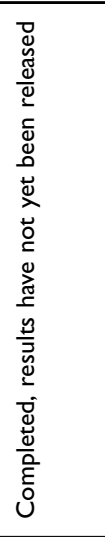 & 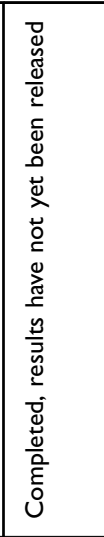 & 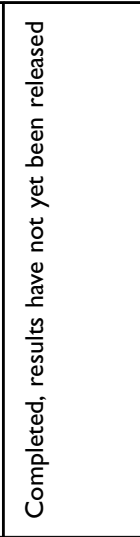 & 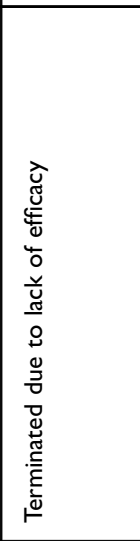 & 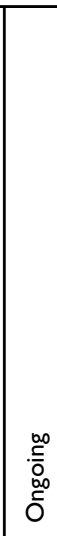 \\
\hline 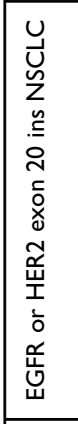 & 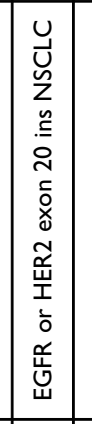 & 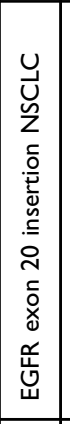 & 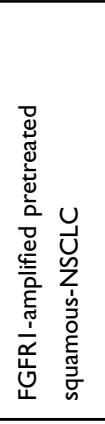 & 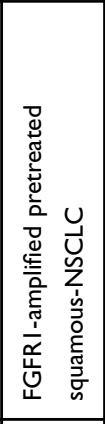 & 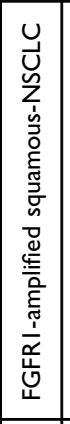 & 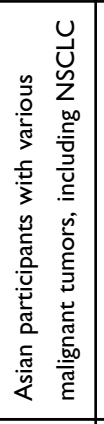 & 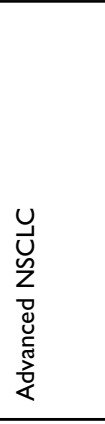 & 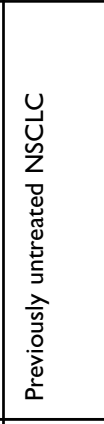 & 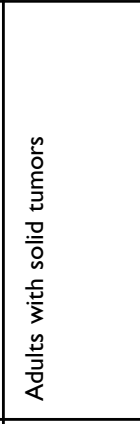 & 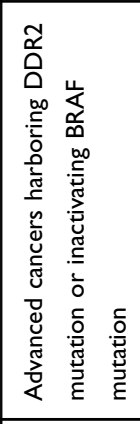 & 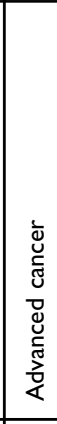 \\
\hline$=$ & $=$ & $\equiv$ & $=$ & $=$ & - & $\stackrel{\underline{\underline{m}}}{ }$ & $\underline{\underline{\prime}}$ & $=$ & - & $=$ & $=$ \\
\hline 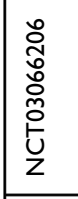 & 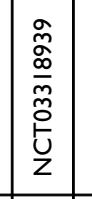 & 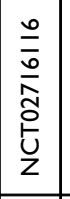 & 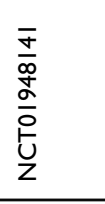 & 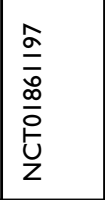 & 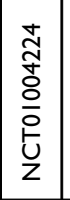 & 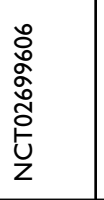 & 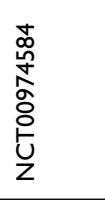 & 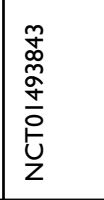 & 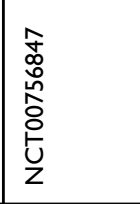 & 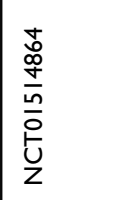 & 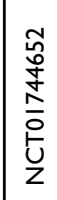 \\
\hline 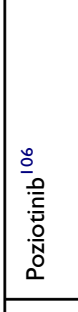 & 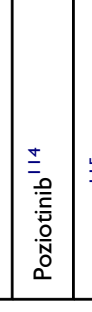 & 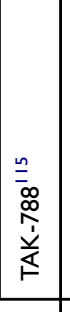 & 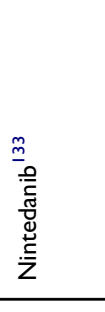 & 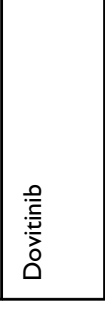 & 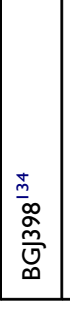 & 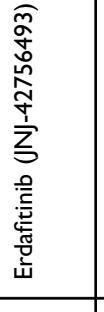 & 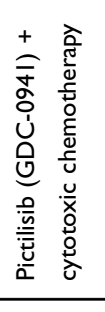 & 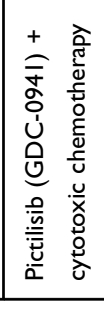 & 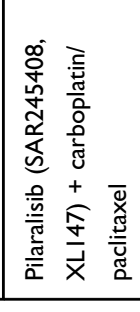 & 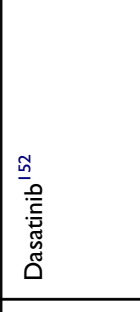 & 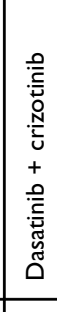 \\
\hline 总 & & & 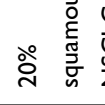 & & & & 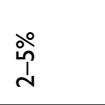 & & & $\stackrel{\circ}{\circ}$ & \\
\hline 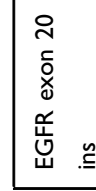 & & & 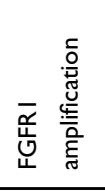 & & & & 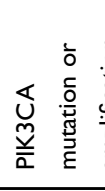 & & & 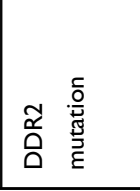 & \\
\hline
\end{tabular}


in patients with ROS1 fusion-positive NSCLC and in patients with secondary mutations resistant to prior TKI. In an ongoing phase I/II trial (TRIDENT-1, NCT03093116), repotrectinib showed an ORR of $82 \%$ in 11 TKI-naive ROS1-positive NSCLC patients, and an ORR of $39 \%$ in 18 patients pretreated with only one prior TKI. Tumor regression occurred in all five patients with G2032R mutation resistant to prior crizotinib treatment. $^{33}$

\section{RET Rearrangements}

The RET (rearranged during transfection) proto-oncogene is located on chromosome 10q11.2, where it encodes a RTK. ${ }^{34}$ RET consists of an extracellular domain, a transmembrane domain, and an intracellular tyrosine kinase. ${ }^{35}$ In NSCLC, at least 12 different gene partners have been described for RET, including KIF5B, CCDC6, NCOA4, MYO5C, EPHA5, TRIM33, CLIP1, ERC1, PICALM, FRMD4A, RUFY2, TRIM24 ${ }^{36}$ with the most frequent fusion partner being KIF5B (72\%). ${ }^{37}$ All RET fusions preserve the tyrosine kinase activity, and each RET partner protein contains a coiled-coil domain, which can promote ligand-independent dimerization and constitutive activation of RET. ${ }^{38}$ Thus, activation of downstream pathways (e.g., JAK/STAT3 and RAS/RAF/MEK/ERK) leads to cellular proliferation, migration, and differentiation. ${ }^{39}$

RET rearrangements can be detected by FISH, NGS, and RT-PCR, but cannot be adequately detected by IHC. ${ }^{40} \mathrm{At}$ present, there is no gold-standard method for the identification of RET rearrangements. RET rearrangements have been observed in approximately 1 to $2 \%$ of NSCLC. ${ }^{41}$ Similar to ROS-1 rearrangements, RET rearrangements in NSCLC are more commonly found among non-smokers or former light smokers less than 60 years of age with adenocarcinoma histology, early lymph node metastases, and advanced disease. ${ }^{41}$ The majority of patients with RET rearrangements have stage IV disease at the time of diagnosis, suggesting that RET-rearranged NSCLC may have a high metastatic potential. ${ }^{37}$ In NSCLC, RET rearrangements are mutually exclusive with other driver mutations, such as ALK or ROS1 rearrangements or EGFR mutations, ${ }^{39,42,43}$ suggesting that RET rearrangements are independent oncogenic drivers in this disease.

\section{Multikinase RET Inhibitors}

Several multikinase inhibitors with nonselective RET inhibitory activity are available for patients with RET-altered cancers (e.g., vandetanib, cabozantinib, lenvatinib, alectinib, and sunitinib) with response rates ranging from $16 \%$ to $53 \%$, and median PFS from 4.5 to 7.3 months. ${ }^{44-48}$ The use of multikinase RET inhibitors has often been associated with a high rate of toxicity due to their activity against VEGFR kinases or EGFR, and their efficacy has been limited. ${ }^{44}$ Although cabozantinib and vandetanib have been recommended for use against RET-rearranged NSCLC by the NCCN guideline outside the context of a clinical trial, no approved standard therapies have been designed to target RET. Novel and potent inhibitors are being developed to selectively target the RET kinase.

Some resistance mechanisms of RET rearrangements are being discovered. Different fusion partners seem to have different therapeutic responses, and KIF5B-RET is associated with lower ORR. Other potential resistance mechanisms include missense mutations in RET, activation of downstream pathway molecules (e.g., ERK, AKT), and the amplification of MDM2. ${ }^{49}$

\section{Selpercatinib (LOXO-292)}

Selpercatinib is a novel, highly selective, ATP-competitive small molecule RET inhibitor that has significant CNS penetration, and a low potential for drug interactions. ${ }^{50}$ It was approved by the FDA for the treatment of advanced RETrearranged NSCLC and medullary thyroid cancers with a breakthrough therapy designation in September 2018. This approval was based on the data from a phase I/II trial (LIBRETTO-001, NCT03157128) reported at the 2018 ASCO meeting, in 30 response-evaluable patients with RET fusion-positive NSCLC, tumor regression occurred regardless of the RET fusion partner, with an ORR of $77 \%$ and another $13 \%$ of the patients experiencing stable disease. ${ }^{51}$ The updated results of this study have been presented at the 2019 WCLC. In 105 previously treated patients, selpercatinib demonstrated an overall ORR of $68 \%$ and a CNS ORR of $91 \%$. The median PFS and DOR was 18.4 months and 20.3 months, respectively. In 34 treatment-naive patients, the ORR was $85 \%$, and the median DOR and PFS were not reached. ${ }^{52}$

\section{BLU-667}

BLU-667 (NCT03037385) is another highly potent and selective small-molecule RET inhibitor that has increased potency and decreased toxicity against RET alterations compared to multikinase inhibitors. ${ }^{53}$ The preliminary analysis of a phase 1 BLU-667 clinical trial for RET-altered NSCLC, medullary thyroid cancers, and other advanced solid tumors was presented at the 2018 American 
Association for Cancer Research (AACR) annual meeting. An ORR of $45 \%$ was observed among the 11 evaluable NSCLC patients, including heavily pretreated patients, who had received prior RET-targeting agents. ${ }^{54}$

\section{MET Activation}

MET (the mesenchymal-to-epithelial transition) is a receptor kinase that activates tyrosine kinases by binding the ligand hepatocyte growth factor (HGF) and inducing MET dimerization and autophosphorylation. ${ }^{55}$ These effects activate downstream signaling pathways, including RAS/RAF/ MAPK, PI3K/AKT/mTOR, WNT/ $\beta$-catenin, and STAT, that play important roles in cell growth, apoptosis, motility, and invasiveness. ${ }^{56,57}$ In NSCLC, several mechanisms of MET activation have been identified, including mutation, rearrangement or amplification of the MET gene, overexpression of MET or HGF protein. ${ }^{55,58}$

\section{MET Exon 14 Skipping Mutation}

$M E T$ exon 14 skipping mutations comprise approximately $3 \%$ of NSCLC cases and are more commonly found in females, elderly patients, non-smokers, pulmonary sarcomatoid carcinoma (PSC), and are associated with poor prognosis. $^{59,60}$ MET exon 14 skipping mutations are mutually exclusive with other known driver genes (e.g., EGFR, KRAS, and HER2 mutations or ALK, ROS1, and RET rearrangements), suggesting that they are independent carcinogenic drivers. ${ }^{61}$ Clinical trials using METtargeted TKIs (e.g., cabozantinib, capmatinib, crizotinib, merestinib, savolitinib, and tepotinib) for NSCLC patients with $M E T$ exon 14 altered-NSCLC are currently ongoing.

\section{Crizotinib}

Crizotinib has been approved for the treatment of $A L K$ positive or ROS1-positive lung cancers and has significant antineoplastic activity in patients with MET alteration. ${ }^{61}$ In the PROFILE 1001 clinical trial, a cohort of 65 patients with $M E T$ exon 14-altered NSCLC were treated with crizotinib and achieved an ORR of $32 \%$, a median PFS of 7.3 months, and a median OS of 20.5 months. ${ }^{62}$ Secondary MET tyrosine kinase domain mutations (e.g., MET D1228 and Y1230) acquired after progression during crizotinib therapy are considered as the emerging mechanisms of resistance to MET inhibition. ${ }^{63-65}$ Subsequent studies found that these mutations confer resistance to type I MET inhibitors which preferentially bind the active conformation of MET (e.g., crizotinib, savolitinib, and capmatinib) through impaired drug binding, while sensitivity to type II inhibitors, which bind the inactive conformation (e.g., glesatinib and cabozantinib), is maintained. ${ }^{66}$

\section{Capmatinib (INC280)}

Another MET-selective agent, capmatinib, has demonstrated a clinically meaningful response rate and a manageable toxicity profile in patients with advanced-stage NSCLC that contain MET exon 14 mutations in an ongoing study (GEOMETRY mono-1, NCT02414139). Results demonstrated an ORR and median PFS of $40.6 \%$ and 5.4 months, respectively, among 69 pretreated patients and $67.9 \%$ and 9.7 months, respectively, for 28 treatment-naïve patients. Preliminary activity in patients with brain metastases was also observed with an intracranial ORR of $54 \%$ in 13 evaluable patients. ${ }^{67}$ Based on these results, the FDA granted breakthrough therapy designation to capmatinib (INC280) as a first-line treatment for patients with metastatic MET exon14 skipping-mutated NSCLC in September 2019.

\section{Tepotinib}

Tepotinib, a MET-selective oral inhibitor, has demonstrated promising antitumor activity in patients with advanced NSCLC that harbor a MET exon 14 skipping mutation and a favorable safety profile with an ORR of $35 \%$ in 41 patients in an ongoing phase II study (VISION, NCT02864992). ${ }^{68}$ Tepotinib was approved by the FDA with a breakthrough therapy designation in September 2019, for the treatment of patients with metastatic NSCLC harboring MET exon 14 skipping alterations who progressed following platinumbased chemotherapy.

\section{Savolitinib}

Savolitinib (AZD6094, HMPL-504, volitinib) is a potent and highly selective inhibitor of MET tyrosine kinase. The preliminary data from a phase II study of savolitinib in MET exon 14 skipping mutant PSC or other types of NSCLC (NCT02897479) were reported at the 2019 AACR Annual Meeting. Savolitinib showed encouraging antitumor activity and an acceptable safety profile. In 31 evaluable patients, the ORR was $51.6 \%$, and the median PFS was not reached. ${ }^{69}$

\section{MET Amplification}

MET gene amplification occurs in 1 to $5 \%$ of treatmentnaïve NSCLC ${ }^{56}$ but more often mediates bypass pathway activation in patients with acquired resistance to EGFRTKIs. The combinations of EGFR- and MET-targeted therapeutics may be effective against these conditions. 


\section{Tepotinib + Gefitinib}

A phase II trial showed that the combination of tepotinib and gefitinib improved the PFS and OS versus chemotherapy in patients with $M E T$-amplified $E G F R$-mutant NSCLC that was resistant to prior EGFR-TKI therapy. The combination of tepotinib and gefitinib in $M E T$ amplification subgroups had an ORR of $66.7 \%$ and a median PFS of 21.2 months whereas chemotherapy had an ORR of $42.9 \%$ and a median PFS of 4.2 months. This combination also had an ORR of $68.4 \%$ and a median PFS of 8.3 months for patients with high MET-expressing tumors compared to $33.3 \%$ and 4.4 months with chemotherapy, respectively. ${ }^{70}$

\section{Savolitinib + Osimertinib}

The TATTON trial is a phase Ib clinical trial that is investigating the clinical response of adding MET inhibitor savolitinib to osimertinib in patients with EGFR-mutant NSCLC that developed resistance to prior EGFR-targeted therapies through $M E T$ gene amplification. The data were presented at the 2019 AACR Annual Meeting. This treatment regimen caused an ORR of $52 \%$ and a median DOR of 7.1 months in 46 patients, who were previously treated with a first- or second-generation EGFR-TKI. The ORR was $28 \%$, and the median DOR was 9.7 months in 48 patients who received a prior third-generation EGFRTKI. $^{71}$ A phase II SAVANNAH trial will further explore the combination in patients with MET-positive disease that has progressed on osimertinib.

\section{BRAF Mutation}

The B-Raf proto-oncoprotein (BRAF) is a serine/threonine kinase that regulates cell proliferation, differentiation, angiogenesis, and cell death. It functions downstream of RAS and signals through the MAPK/ERK pathway. ${ }^{72}$ $B R A F$ mutations appear in approximately 2 to $4 \%$ of NSCLC and are more commonly found in current or former smokers and female patients. V600E is the most common mutation and accounts for 1 to $2 \%$ of lung adenocarcinomas and roughly $50 \%$ of $B R A F$-mutant NSCLC. Compared with non-V600E genotypes, V600E is associated with more aggressive tumor histology and a poorer prognosis. ${ }^{73,74}$ BRAF mutations are also commonly found in melanoma with a prevalence of $50 \%$, and $\mathrm{V} 600 \mathrm{E}$ is the most common mutation. Targeting BRAF has made some progress in the treatment of melanoma. Single-agent BRAF inhibitors (vemurafenib, dabrafenib) and the combination of dabrafenib and the mitogenactivated protein kinase (MEK) inhibitor, trametinib, have already been approved by the FDA for metastatic $B R A F$ V600E-mutant melanoma and are being explored for BRAF-mutated NSCLC. ${ }^{75}$

A retrospective study EURAF evaluated the efficacy of different BRAF inhibitors, including vemurafenib, dabrafenib, and sorafenib, against advanced NSCLC harboring $B R A F$ mutations. The PFS and median OS for BRAF inhibition therapy were five months and 10.2 months, respectively. ${ }^{76}$ In chemotherapy-pretreated patients with NSCLC harboring BRAF V600E, the combination of dabrafenib and trametinib had an ORR of $63 \%$, median PFS of 9.7 months, and median OS of 18.2 months. In treatment-naïve BRAF V600E-mutant metastatic NSCLC, this combination showed an ORR of $64 \%$, median PFS of 10.9 months, and median OS of 24.6 months compared to an ORR of $33 \%$, median PFS of 5.5 months, and median OS of 12.7 months for dabrafenib monotherapy. ${ }^{77-79}$ Based on these results, the combination of dabrafenib and trametinib was approved in June 2017 by the regulatory authorities of both the US and European Union for treatment of advanced NSCLC harboring the BRAF V600E mutation regardless of the previous therapy. ${ }^{80}$ Belvarafenib, a panRAF kinase inhibitor, was well-tolerated and exhibited antitumor activity in patients with advanced solid tumors harboring RAS or RAF mutations, and further investigation of its combination with the MEK inhibitor cobimetinib is ongoing (NCT02405065, NCT03118817). ${ }^{81}$

Several possible secondary resistance mechanisms to BRAF inhibitors have been proposed, including reactivation of ERK signaling through the MAPK pathway, bypassing of the MAPK pathway via the activation of alternative signaling pathways and other uncharacterized mechanisms. ${ }^{82}$ The secondary resistance mechanism to the dual inhibition is more complex and requires further investigation.

\section{NTRK Rearrangements}

The neurotrophic tropomyosin receptor kinase (NTRK) genes (NTRK1, NTRK2, and NTRK3) encode three TRK proteins (TRKA, TRKB, and TRKC), which play an important role in the cell growth, differentiation, and apoptosis of peripheral and central nervous system neurons. ${ }^{83}$ They activate downstream PI3K/AKT/mTOR, RAS/RAF/MAPK, PLC- $\gamma$, and protein kinase C pathways to control cell cycle progression, proliferation, apoptosis, and survival. ${ }^{84-87}$ NTRK1 and NTRK2 rearrangements occur in 3 to $4 \%$ NSCLC, ${ }^{88,89}$ and CD74, MPRIP, SQSTM1, TRIM24 are their known fusion partners. ${ }^{90}$ 
The clinical and pathologic features of patients with NTRK1 and NTRK2 rearrangements are not well characterized.

\section{Larotrectinib (LOXO-I0I)}

Larotrectinib (LOXO-101) is a highly selective pan-TRK inhibitor. $^{91}$ In an ongoing study of 55 patients with a variety of NTRK fusion-positive cancers, the ORR is $75 \%$ regardless of tumor type. ${ }^{92}$ The results for four NSCLC patients enrolled in this study were reported at the 2018 WCLC. At the time of analysis, three of the four patients had ongoing responses to the drug (ranging from 5.7 to 12 months) whereas one patient had stable disease that finally progressed after 300 days of treatment. ${ }^{93}$ The success of this trial led to FDA approval of larotrectinib for adult and pediatric patients with solid tumors harboring NTRK gene fusions in May $2018^{94}$ Three different categories of mutations were observed after larotrectinib progression and may represent resistance mechanisms, including substitutions in the solvent front (NTRK1 p. G595R, NTRK3 p.G623R), the gatekeeper position (NTRK1 p.F589L), and the xDFG position (NTRK1 p. G667S, NTRK3 p.G696A). ${ }^{92}$

\section{LOXO- 195}

LOXO-195 is a second-generation TRK-selective inhibitor that was developed to overcome NTRK1 p.G595Rmediated resistance. ${ }^{95}$ It has been used in two patients with NTRK fusion-positive cancer (colon and infantile fibrosarcoma) that progressed after larotrectinib treatment. Both patients achieved objective responses from LOXO195 therapy. ${ }^{95}$ Furthermore, a multicenter phase $1 / 2$ clinical trial of LOXO-195 in patients with solid tumors harboring NTRK fusion is now underway (NCT03215511).

\section{Entrectinib (RXDX-I0I)}

The multi-kinase inhibitor entrectinib also showed antiNTRK activity. The integrated analysis of STARTRK-1, STARTRK-2, and ALKA-372-001 trials we mentioned in ROS1 rearrangements also reported the efficacy of entrectinib in NTRK fusion-positive solid tumors. In 54 patients, it showed an ORR of $57.4 \%$, a median PFS of 11.2 months, and a median OS of 20.9 months. ${ }^{96}$ It was recommended by the NCCN Guidelines for the treatment of advanced NTRK fusion-positive NSCLC, and approved by FDA with breakthrough therapy designation. ${ }^{27}$

\section{HER2 Mutation}

As a membrane-bound tyrosine kinase in the ERBB family, human epidermal growth factor receptor 2 (HER2 or ERBB2) differs from EGFR (ERBB1) due to its lack of endogenous ligand. HER2 activates signal transduction pathways, including PI3K, MAPK, and JAK/STAT, through heterodimerization with other members of the ERBB family, which promotes cell proliferation and survival. ${ }^{97}$ In NSCLC, HER2 amplification is not considered to be oncogenic driver mutation, but one of the secondary mechanisms of resistance to EGFR-TKIs. ${ }^{98}$ HER2 mutation is regarded as potential drivers of oncogenesis which have been identified in approximately 2 to $4 \%$ of NSCLC and are associated with adenocarcinoma histology, female gender, Asian ethnicity, and neversmoked status, and the YVMA 776-779 ins in exon 20 is the most frequent HER2 mutation. ${ }^{99}$

HER2-targeted therapies have had great success against breast cancer, including trastuzumab, pertuzumab, ado-trastuzumab emtansine (T-DM1), and lapatinib, which have been approved by the FDA for HER2-positive breast cancer. $^{100}$ However, currently available targeted drugs have limited activity in HER2-mutant NSCLC, and there are no HER2-targeted drugs approved for NSCLC. ${ }^{101}$ A retrospective international multicenter study analyzed 27 patients with stage IV or recurrent HER2-mutant lung adenocarcinoma treated with afatinib and found a median time-to-treatment failure (TTF) of three months, and a median DOR of six months. ${ }^{102}$ The responses appeared to be better in the exon 20 ins subgroups. A recent study of afatinib in heavily pretreated NSCLC patients showed a median TTF of 9.6 months, an ORR of $33 \%$, and a DCR of $100 \%$ among ten patients with HER2 exon 20 ins. ${ }^{103}$ In a retrospective study of 101 NSCLC patients with HER2 mutations, for 29 patients who received neratinib, lapatinib or afatinib, the ORR was 7.4\%, and median PFS was 3.4 months; for 58 patients who received T-DM1 alone or trastuzumab in combination with chemotherapy, the ORR was 50.9\%, and median PFS was 4.8 months. ${ }^{101}$ A phase II basket trial assessed the activity of T-DM1 in 18 HER2-mutant NSCLC patients and showed an ORR of $44 \%$ with a median PFS of 5 months. ${ }^{104}$ Poziotinib showed promising preclinical and early clinical activity in NSCLC patients with HER2 or EGFR exon 20 ins, $^{105}$ in an ongoing phase II study (NCT03066206), initial responses of 50\% and a median PFS of 5.1 months were observed in 12 evaluable 
advanced HER2 exon 20 ins NSCLC patients. ${ }^{106}$ An ongoing phase I study of another novel HER2-targeted drug, trastuzumab deruxtecan (DS-8201a), demonstrated an ORR of $62.5 \%$ and DCR of $75 \%$ in 12 patients with HER2-expressing or -mutated NSCLC. ${ }^{107}$ A phase II study for this drug against the same condition recently began accrual (NCT03505710).

The resistance mechanisms upon progression on HER2 targeted therapies are not fully understood. A recent study of trastuzumab in combination with chemotherapy or afatinib in 9 patients with HER2-mutant metastatic lung adenocarcinoma suggested that PIK3CA mutation and increased HER2 copy number may be potential resistance mechanisms. ${ }^{108}$

\section{EGFR Exon 20 Ins}

EGFR exon 20 ins mutations occur in about $1.8 \%$ of all NSCLC and $12 \%$ of cases with EGFR mutants. ${ }^{109}$ Similar to classical activating EGFR mutations, EGFR exon 20 ins mutations are enriched in women, non-smokers, Asian populations, and tumors with adenocarcinoma histology. ${ }^{110}$ EGFR exon 20 ins patients respond poorly to targeted EGFR inhibitors, including the third-generation inhibitor, osimertinib. $^{111-113}$

\section{Poziotinib}

Poziotinib is a potent and clinically active inhibitor of EGFR and HER2 exon 20 ins. $^{105}$ In a phase II trial for cancers containing these mutations, poziotinib treatment resulted in an ORR of $55 \%$ and a median PFS of 5.5 months for 44 advanced NSCLC patients with EGFR exon 20 ins. Responses were also observed in $62 \%$ of TKI-refractory patients (NCT03066206). ${ }^{106}$ Although the FDA refused to grant breakthrough therapy designation to poziotinib for the treatment of patients with metastatic NSCLC containing EGFR exon 20 mutations, the preliminary results of poziotinib from an ongoing phase II clinical trial (NCT03318939) demonstrated an ORR of $40 \%$ and a median DOR of 6.6 months. ${ }^{114}$

\section{TAK-788}

TAK-788 is an oral inhibitor with potent, selective preclinical activity against EGFR/HER2, including exon 20 ins. Results from a Phase 1/2 (NCT02716116) showed TAK788 yielded a median PFS of 7.3 months and an ORR of $43 \%$ in 28 patients with locally advanced or metastatic NSCLC with EGFR exon 20 ins. In a patient subgroup without brain metastases at baseline, the ORR was 56\% (n $=9 / 16)$, and the median PFS was 8.1 months. ${ }^{115}$

\section{NRG I Fusion}

The neuregulin 1 gene (NRG1) is located on chromosome 10q23.1. ${ }^{116} N R G 1$ fusion is a novel driver gene identified in many cancer types. ${ }^{117}$ It can induce the expression of the extracellular EGF-like domain of NRG1, which binds to HER3 (ERBB3), thereby stimulating heterodimerization of HER3 with HER2, and subsequently actives the AKT and MAPK pathways. ${ }^{118}$

$N R G 1$ fusion occurs in $1-2 \%$ of NSCLC, ${ }^{119}$ and is mutually exclusive with other oncogenic alterations. ${ }^{120} \mathrm{It}$ is mainly identified using RNA-based assays. CD74 and SCLA3A2 are the most common upstream partners, and other partners include SDC4, SLC3A2, TNC, MDK, ATP1B1, DIP2B, RBPMS, MRPL13, ROCK1, DPYSL2, and PARP8. ${ }^{117}$ NRG1 fusion is more common in women, never smokers and adenocarcinoma histology, especially mucinous subtypes. ${ }^{117,121}$

Based on the activation mechanism, targeting the HER2/HER3 signaling pathway is a possible therapeutic strategy for NRG1 fusion. The pan-ERBB inhibitor afatinib has been reported to have anti-tumor activity in several patients with NSCLC harboring NRG1 fusion. In 2017, two patients with NRG1 fusion-positive stage IV NSCLC were reported to have a durable clinical response to afatinib of 10 and 12 months, respectively. ${ }^{122}$ Another report presented at the 2019 WCLC showed four NSCLC patients with $N R G 1$ gene fusion achieved a certain degree of response or tumor stabilization when treated with afatinib. ${ }^{123}$ Response to anti-ERBB3 monoclonal antibody GSK2849330 has also been reported. ${ }^{120}$ Further studies are needed to explore targeted therapies for NRG1 fusion.

\section{FGFRI Amplification}

FGFR1 (fibroblast growth factor receptor 1) belongs to a family of four transmembrane tyrosine kinase receptors (FGFR1-4) that regulates angiogenesis, embryogenesis, inflammation, and malignant tumor cell proliferation through the downstream activation of the RAS/RAF/MAPK, PI3K/ AKT/mTOR, STAT, and PLC $\gamma$ pathways. ${ }^{124,125}$ The FGFR1 gene, located on chromosome $10 \mathrm{q}$, is amplified in about $20 \%$ of squamous NSCLC, more common in males and active smokers, and may be a negative prognostic marker in earlystage NSCLC patients treated with surgery. ${ }^{126}$ A 3.5-fold amplification of this gene was recognized as the distribution cut-off for patient survival and may represent a stratification 
factor for clinical trials. ${ }^{127-129}$ In addition, a recent study demonstrated that FGFR mutations might increase the risk of lymph node metastasis in squamous NSCLC and be an independent predictive factor for inferior survival. ${ }^{130}$ There are currently two types of FGFR inhibitors: 1) molecules directed against the FGFR domain, which are selective FGFR TKIs; 2) multitarget inhibitors that are nonselective FGFR TKIs.

\section{Nonselective FGFR TKIs}

Clinical trials using nonselective FGFR TKIs (e.g., lucitanib, lenvatinib, dovitinib, nintedanib, ponatinib, cediranib, pazopanib, regorafenib, and brivanib) have demonstrated limited activity and undesired side toxicities, such as hypertension, proteinuria, cardiovascular events, and hypothyroidism due to VEGF inhibition. ${ }^{131,132}$ Nintedanib is the only FGFR inhibitor approved by the European Medical Agency (EMA) for second-line treatment in combination with docetaxel for NSCLC patients with locally advanced, recurrent, or metastatic disease and adenocarcinoma histology. ${ }^{132}$ In a phase II trial of nintedanib in patients with FGFRl-amplified pretreated squamous NSCLC, a 6-month PFS was observed for the six FGFR1-amplified patients (NCT01948141). ${ }^{133}$ A phase II trial for another nonselective FGFR TKI, dovitinib, is ongoing in pretreated squamous NSCLC patients with FGFR1 amplification (NCT01861197).

\section{Selective FGFR TKIs}

Clinical trials with selective FGFR TKIs, including BGJ398, AZD4547, debio-1347, LY2874455, ARQ-087, erdafitinib, and TAS-120, have had a range of activity. ${ }^{131,132}$ An ORR of $11.1 \%$ and DCR of $50 \%$ were reported for 36 FGFR1amplified squamous NSCLC patients treated with BGJ398. ${ }^{134}$ Erdafitinib (JNJ-42756493) has demonstrated antitumor activity against urothelial carcinoma. In April 2019, it received accelerated approval from the FDA for the treatment of adults with locally advanced or metastatic urothelial carcinoma with FGFR3 or FGFR2 genetic alterations, who have progressed during or following at least one line of prior platinum-containing chemotherapy, including within 12 months of neoadjuvant or adjuvant platinum-containing chemotherapy. ${ }^{135}$ A phase IIa study of erdafitinib in Asian participants with various malignant tumors, including NSCLC, is ongoing (NCT02699606).

\section{PI3KCA Mutation or Amplification}

Phosphatidylinositol-3 kinases (PI3K) belong to a family of heterodimeric kinases, which play an important role in the regulation of cell growth, survival, and motility. There are three classes of PI3K (I, II, and III), which impact cellular signal transduction in different roles. Phosphatidylinositol 3-kinase, catalytic (PI3KCA) is a catalytic subunit of the class IA PI3K. Aberrant activation of PI3KCA through gene mutation or amplification is associated with human carcinogenesis. ${ }^{136}$ PI3KCA can also be activated by upstream growth factor receptors followed by subsequent activation of its downstream pathways, including AKT/ mTORC1/p70S6K. Tumor suppressor PTEN is a key negative regulator of $\mathrm{PI} 3 \mathrm{~K} / \mathrm{AKT} / \mathrm{mTOR}$ activation through PIK3CA. ${ }^{137,138}$

$P I 3 K C A$ amplification is more common in squamous NSCLC (33 to 37\%) than adenocarcinoma (5 to 6\%). ${ }^{139,140}$ PI3KCA mutations occur in about 2 to $5 \%$ NSCLC and are also more prevalent in squamous NSCLC. ${ }^{139-141}$ In adenocarcinoma, these mutations are associated with a poorer prognosis, ${ }^{142}$ have been reported to be concurrent with other oncogenic drivers and may be related to resistance mechanisms against TKIs because they have also been found in EGFR-mutant NSCLC that has developed acquired resistance to EGFR-TKIs. ${ }^{143,144}$

The pan PI3K inhibitor Buparlisib (BKM120) did not demonstrate sufficient clinical activity in patients with relapsed NSCLC with $P I 3 K C A$ mutation in a phase II study but had activity when combined with the mTOR inhibitor everolimus in NSCLC preclinical models. It also had activity in pretreated head and neck squamous cell carcinoma patients when combined with paclitaxel in a phase II study. ${ }^{144-146}$ These trials suggest that the antitumor activity of a single PI3KCA-targeted agent may be limited, but combined therapy may provide better results. Additional clinical trials with other PI3K inhibitors combined with targeted agents and/or cytotoxic chemotherapy, such as pictilisib (GDC-0941) in combination with cytotoxic chemotherapy (e.g., paclitaxel, carboplatin, pemetrexed, and cisplatin) (NCT00974584, NCT01493843); pilaralisib (SAR245408, XL147) combined with carboplatin/paclitaxel (NCT00756847) have been completed, but the results have not yet been released.

\section{DDR2 Mutation}

Activating discoidin domain receptor 2 gene (DDR2) mutations have mainly been identified in approximately $4 \%$ of 
squamous NSCLC. These mutations can promote carcinogenesis through cell migration and proliferation. ${ }^{147,148}$ Dasatinib and its combination with erlotinib have demonstrated activity in NSCLC patients with DDR2 mutations. ${ }^{148-150}$ However, several dasatinib clinical trials were terminated prematurely due to intolerable toxicities, lack of efficacy, and slow accrual. $^{151,152}$ Because excessive toxicity could hinder the potential benefit of this drug, an ongoing phase II trial (NCT01744652) is currently trying to identify the highest tolerable drug doses for the combination of dasatinib and crizotinib that can be given to patients with advanced cancer.

\section{Conclusion}

With the rapid development of precision medicine over the past decade, many oncogenic drivers have been discovered, and a paradigmatic change has occurred in the diagnosis and treatment of patients with advanced NSCLC. EGFR- and ALK-targeted therapy has become standard therapy for patients with these mutations. In this review, we discussed the rare driver genes in NSCLC, focusing on the clinical characteristics, currently approved therapies, and resistance mechanisms. Although targeted therapies have been greatly successful in recent years, the benefits obtained are limited due to the inevitable development of drug resistance. To improve efficacy, overcome resistance, and minimize toxicity, further studies are needed to explore the resistance mechanisms and discover more effective targeted therapies.

\section{Acknowledgment}

This study was supported by grants from the National Natural Science Foundation of China (grant no. 81501990).

\section{Disclosure}

The authors report no conflicts of interest in this work.

\section{References}

1. Nagarajan L, Louie E, Tsujimoto Y, Balduzzi PC, Huebner K, Croce CM. The human c-ros gene (ROS) is located at chromosome region 6q16-6q22. Proc Natl Acad Sci U S A. 1986;83 (17):6568-6572. doi:10.1073/pnas.83.17.6568

2. Satoh H, Yoshida MC, Matsushime H, Shibuya M, Sasaki M. Regional localization of the human c-ros-1 on 6q22 and flt on 13q12. Jpn J Cancer Res. 1987;78(8):772-775.

3. Acquaviva J, Wong R, Charest A. The multifaceted roles of the receptor tyrosine kinase ROS in development and cancer. Biochim Biophys Acta. 2009;1795(1):37-52. doi:10.1016/j.bbcan.2008.07.006

4. Gainor JF, Shaw AT. Novel targets in non-small cell lung cancer: ROS1 and RET fusions. Oncologist. 2013;18(7):865-875. doi:10.1634/theoncologist.2013-0095
5. Charest A, Wilker EW, McLaughlin ME, et al. ROS fusion tyrosine kinase activates a SH2 domain-containing phosphatase-2/phosphatidylinositol 3-kinase/mammalian target of rapamycin signaling axis to form glioblastoma in mice. Cancer Res. 2006;66 (15):7473-7481. doi:10.1158/0008-5472.CAN-06-1193

6. Davies KD, Le AT, Theodoro MF, et al. Identifying and targeting ROS1 gene fusions in non-small cell lung cancer. Clin Cancer Res. 2012;18(17):4570-4579. doi:10.1158/1078-0432.CCR-12-0550

7. Jun HJ, Johnson H, Bronson RT, de Feraudy S, White F, Charest A. The oncogenic lung cancer fusion kinase CD74-ROS activates a novel invasiveness pathway through E-Syt1 phosphorylation. Cancer Res. 2012;72(15):3764-3774. doi:10.1158/0008-5472. CAN-11-3990

8. Lin JJ, Shaw AT. Recent advances in targeting ROS1 in lung cancer. J Thorac Oncol. 2017;12(11):1611-1625. doi:10.1016/j. jtho.2017.08.002

9. Li Z, Shen L, Ding D, et al. Efficacy of crizotinib among different types of ROS1 fusion partners in patients with ROS1-rearranged non-small cell lung cancer. J Thorac Oncol. 2018;13(7):987-995. doi:10.1016/j.jtho.2018.04.016

10. Lin JJ, Ritterhouse LL, Ali SM, et al. ROS1 fusions rarely overlap with other oncogenic drivers in non-small cell lung cancer. J Thorac Oncol. 2017;12(5):872-877. doi:10.1016/j. jtho.2017.01.004

11. Shaw AT, Ou SH, Bang YJ, et al. Crizotinib in ROS1-rearranged non-small-cell lung cancer. $N$ Engl $J$ Med. 2014;371 (21):1963-1971. doi:10.1056/NEJMoa1406766

12. Bergethon K, Shaw AT, Ou SH, et al. ROS1 rearrangements define a unique molecular class of lung cancers. J Clin Oncol. 2012;30 (8):863-870. doi:10.1200/JCO.2011.35.6345

13. Shaw AT, Kim DW, Nakagawa K, et al. Crizotinib versus chemotherapy in advanced ALK-positive lung cancer. $N$ Engl $J$ Med. 2013;368(25):2385-2394. doi:10.1056/NEJMoa1214886

14. Solomon BJ, Mok T, Kim DW, et al. First-line crizotinib versus chemotherapy in ALK-positive lung cancer. $N$ Engl J Med. 2014;371(23):2167-2177. doi:10.1056/NEJMoa1408440

15. Moro-Sibilot D, Faivre L, Zalcman G, et al. Crizotinib in patients with advanced ROS1-rearranged non-small cell lung cancer (NSCLC). Preliminary results of the ACSé phase II trial. J Clin Oncol. 2015;33 (15_suppl):8065. doi:10.1200/jco.2015.33.15_suppl.8065

16. Mazieres J, Zalcman G, Crino L, et al. Crizotinib therapy for advanced lung adenocarcinoma and a ROS1 rearrangement: results from the EUROS1 cohort. J Clin Oncol. 2015;33(9):992-999. doi: $10.1200 / J C O .2014 .58 .3302$

17. NCCN Clinical Practice Guidelines in Oncology (NCCN Guidelines). Non-small cell lung cancer. Version 2.2017. Available from: www.nccn.org. Published 2017. Accessed April 4, 2019 ..

18. Gainor JF, Tseng D, Yoda S, et al. Patterns of metastatic spread and mechanisms of resistance to crizotinib in ROS1-positive non-smallcell lung cancer. JCO Precis Oncol. 2017;1:1-13.

19. Awad MM, Katayama R, McTigue M, et al. Acquired resistance to crizotinib from a mutation in CD74-ROS1. $N$ Engl $J$ Med. 2013;368(25):2395-2401. doi:10.1056/NEJMoa1215530

20. Song A, Kim TM, Kim DW, et al. Molecular changes associated with acquired resistance to crizotinib in ROS1-rearranged non-small cell lung cancer. Clin Cancer Res. 2015;21 (10):2379-2387. doi:10.1158/1078-0432.CCR-14-1350

21. Drilon A, Somwar R, Wagner JP, et al. A novel crizotinib-resistant solvent-front mutation responsive to cabozantinib therapy in a patient with ROS1-rearranged lung cancer. Clin Cancer Res. 2016;22(10):2351-2358. doi:10.1158/1078-0432.CCR-15-2013

22. McCoach CE, Le AT, Gowan K, et al. Resistance mechanisms to targeted therapies in $\operatorname{ROS1}(+)$ and $\operatorname{ALK}(+)$ non-small cell lung cancer. Clin Cancer Res. 2018;24(14):3334-3347. doi:10.1158/ 1078-0432.CCR-17-2452 
23. Lim SM, Kim HR, Lee JS, et al. Open-label, multicenter, phase II study of ceritinib in patients with non-small-cell lung cancer harboring ROS1 rearrangement. $J$ Clin Oncol. 2017;35 (23):2613-2618. doi:10.1200/JCO.2016.71.3701

24. Ardini E, Menichincheri M, Banfi P, et al. Entrectinib, a Pan-TRK, ROS1, and ALK Inhibitor with activity in multiple molecularly defined cancer indications. Mol Cancer Ther. 2016;15 (4):628-639. doi:10.1158/1535-7163.MCT-15-0758

25. Menichincheri M, Ardini E, Magnaghi $P$, et al. Discovery of entrectinib: a new 3-aminoindazole as a potent Anaplastic Lymphoma Kinase (ALK), c-ros oncogene 1 kinase (ROS1), and pan-tropomyosin receptor kinases (Pan-TRKs) inhibitor. $J$ Med Chem. 2016;59(7):3392-3408. doi:10.1021/acs.jmedchem.6b00064

26. Doebele RC, Ahn MJ, Siena S, et al. Efficacy and safety of entrectinib in locally advanced or metastatic ROS1-positive Non-Small Cell Lung Cancer (NSCLC). In: IASLC 19th World Conference on Lung Cancer; 2018. Toronto, Canada.

27. NCCN Clinical Practice Guidelines in Oncology (NCCN Guidelines). Non-small cell lung cancer. Version 7.2019. Available from: www.nccn. org. Published 2019. Accessed October 4, 2019.

28. Facchinetti F, Loriot Y, Kuo MS, et al. Crizotinib-resistant ROS1 mutations reveal a predictive kinase inhibitor sensitivity model for ROS1- and ALK-rearranged lung cancers. Clin Cancer Res. 2016;22(24):5983-5991. doi:10.1158/1078-0432.CCR-16-0917

29. Zou HY, Li Q, Engstrom LD, et al. PF-06463922 is a potent and selective next-generation ROS1/ALK inhibitor capable of blocking crizotinib-resistant ROS1 mutations. Proc Natl Acad Sci US A. 2015;112(11):3493-3498. doi:10.1073/pnas.1420785112

30. Shaw AT, Felip E, Bauer TM, et al. Lorlatinib in non-small-cell lung cancer with ALK or ROS1 rearrangement: an international, multicentre, open-label, single-arm first-in-man phase 1 trial. Lancet Oncol. 2017;18 (12):1590-1599. doi:10.1016/S1470-2045(17)30680-0

31. A study of PF-06463922 an ALK/ROS1 inhibitor in patients with advanced non small cell lung cancer with specific molecular alterations. Available from: https:/clinicaltrials.gov/ct2/show/ results/NCT01970865. Accessed April 4, 2019.

32. Fujiwara Y, Takeda M, Yamamoto N, et al. Safety and pharmacokinetics of DS-6051b in Japanese patients with non-small cell lung cancer harboring ROS1 fusions: a phase I study. Oncotarget. 2018;9(34):23729-23737. doi:10.18632/oncotarget.v9i34

33. Cho BC, Drilon AE, Doebele RC, et al. Safety and preliminary clinical activity of repotrectinib in patients with advanced ROS1 fusion-positive non-small cell lung cancer (TRIDENT-1 STUDY). J Clin Oncol. 2019: 9011.

34. Ishizaka $\mathrm{Y}$, Itoh $\mathrm{F}$, Tahira $\mathrm{T}$, et al. Human ret proto-oncogene mapped to chromosome 10q11.2. Oncogene. 1989;4(12):1519-1521.

35. Eng C. RET proto-oncogene in the development of human cancer. J Clin Oncol. 1999;17(1):380-393. doi:10.1200/JCO.1999.17.1.380

36. Ferrara R, Auger N, Auclin E, Besse B. Clinical and translational implications of RET rearrangements in non-small cell lung cancer. $J$ Thorac Oncol. 2018;13(1):27-45. doi:10.1016/j.jtho.2017.10.021

37. Gautschi O, Milia J, Filleron T, et al. Targeting RET in patients with RET-rearranged lung cancers: results from the global, multicenter RET registry. J Clin Oncol. 2017;35(13):1403-1410. doi:10.1200/JCO.2016.70.9352

38. Soda M, Choi YL, Enomoto M, et al. Identification of the transforming EML4-ALK fusion gene in non-small-cell lung cancer Nature. 2007;448(7153):561-566. doi:10.1038/nature05945

39. Kohno T, Ichikawa H, Totoki Y, et al. KIF5B-RET fusions in lung adenocarcinoma. Nat Med. 2012;18(3):375-377. doi:10.1038/ nm.2644

40. Go H, Jung YJ, Kang HW, et al. Diagnostic method for the detection of KIF5B-RET transformation in lung adenocarcinoma. Lung Cancer. 2013;82(1):44-50. doi:10.1016/j.lungcan.2013.07.009
41. Wang R, Hu H, Pan Y, et al. RET fusions define a unique molecular and clinicopathologic subtype of non-small-cell lung cancer. J Clin Oncol. 2012;30(35):4352-4359. doi:10.1200/JCO.2012.44.1477

42. Lipson D, Capelletti M, Yelensky R, et al. Identification of new ALK and RET gene fusions from colorectal and lung cancer biopsies. Nat Med. 2012;18(3):382-384. doi:10.1038/nm.2673

43. Takeuchi K, Soda M, Togashi Y, et al. RET, ROS1 and ALK fusions in lung cancer. Nat Med. 2012;18(3):378-381. doi:10.1038/nm.2658

44. Mendoza L. Clinical development of RET inhibitors in RET-rearranged non-small cell lung cancer: update. Oncol Rev. 2018;12(2):352.

45. Drilon A, Rekhtman N, Arcila M, et al. Cabozantinib in patients with advanced RET-rearranged non-small-cell lung cancer: an open-label, single-centre, phase 2, single-arm trial. Lancet Oncol. 2016;17(12):1653-1660. doi:10.1016/S1470-2045(16)30562-9

46. Yoh K, Seto T, Satouchi M, et al. Vandetanib in patients with previously treated RET-rearranged advanced non-small-cell lung cancer (LURET): an open-label, multicentre phase 2 trial. Lancet Respir Med. 2017;5 (1):42-50. doi:10.1016/S2213-2600(16)30322-8

47. Dutcus CE, Nokihara H, Yang JC, et al. Phase 2 study of lenvatinib (LN) in patients (Pts) with RET fusion-positive adenocarcinoma of the lung. Ann Oncol. 2016;27(suppl_6). doi:10.1093/annonc/ mdw 141

48. Lee SH, Lee JK, Ahn MJ, et al. Vandetanib in pretreated patients with advanced non-small cell lung cancer-harboring RET rearrangement: a phase II clinical trial. Ann Oncol. 2017;28(2):292-297. doi:10.1093/annonc/mdw559

49. Bronte G, Ulivi P, Verlicchi A, Cravero P, Delmonte A, Crinò L. Targeting RET-rearranged non-small-cell lung cancer: future prospects. Lung Cancer (Auckl). 2019;10:27-36. doi:10.2147/ LCTT.S192830

50. Subbiah V, Velcheti V, Tuch BB, et al. Selective RET kinase inhibition for patients with RET-altered cancers. Ann Oncol. 2018;29(8):1869-1876. doi:10.1093/annonc/mdy137

51. Dolgin E. LOXO-292 reins in RET-driven tumors. Cancer Discov. 2018;8(8):904-905.

52. Drilon A, Oxnard G, Wirth L, et al. Registrational results of LIBRETTO-001: a phase 1/2 trial of selpercatinib (LOXO-292) in patients with RET fusion-positive lung cancers. 2019 international association for the study of lung cancer world conference on lung cancer; September 7 to 10, 2019; Barcelona, Spain. J Thorac Oncol. 2019;14:S6-S7. doi:10.1016/j.jtho.2019.08.059

53. Subbiah V, Gainor JF, Rahal R, et al. Precision targeted therapy with BLU-667 for RET-driven cancers. Cancer Discov. 2018;8 (7):836-849. doi:10.1158/2159-8290.CD-18-0338

54. Subbiah V, Taylor M, Lin J, et al. Abstract CT043: Highly potent and selective RET inhibitor, BLU-667, achieves proof of concept in a phase I study of advanced, RET-altered solid tumors. Cancer research. 2018;78 (13 Supplement):CT043-CT043.

55. Cipriani NA, Abidoye OO, Vokes E, Salgia R. MET as a target for treatment of chest tumors. Lung Cancer. 2009;63(2):169-179. doi:10.1016/j.lungcan.2008.06.011

56. Drilon A, Cappuzzo F, Ou SI, Camidge DR. Targeting MET in lung cancer: will expectations finally be MET? J Thorac Oncol. 2017;12 (1):15-26. doi:10.1016/j.jtho.2016.10.014

57. Frampton GM, Ali SM, Rosenzweig M, et al. Activation of MET via diverse exon 14 splicing alterations occurs in multiple tumor types and confers clinical sensitivity to MET inhibitors. Cancer Discov. 2015;5(8):850-859. doi:10.1158/2159-8290.CD-15-0285

58. Gelsomino F, Facchinetti F, Haspinger ER, et al. Targeting the MET gene for the treatment of non-small-cell lung cancer. Crit Rev Oncol Hematol. 2014;89(2):284-299. doi:10.1016/j.critrev onc. 2013.11 .006 
59. Tong JH, Yeung SF, Chan AW, et al. MET amplification and exon 14 splice site mutation define unique molecular subgroups of non-small cell lung carcinoma with poor prognosis. Clin Cancer Res. 2016;22(12):3048-3056. doi:10.1158/1078-0432. CCR-15-2061

60. Vuong HG, Ho ATN, Altibi AMA, Nakazawa T, Katoh R, Kondo T. Clinicopathological implications of MET exon 14 mutations in non-small cell lung cancer - A systematic review and meta-analysis. Lung Cancer. 2018;123:76-82. doi:10.1016/j.lungcan.2018.07.006

61. Cortot AB, Kherrouche Z, Descarpentries C, et al. Exon 14 deleted MET receptor as a new biomarker and target in cancers. $J$ Natl Cancer Inst. 2017;109:5. doi:10.1093/jnci/djw262

62. Drilon A, Clark J, Weiss J, et al. OA12.02 updated antitumor activity of crizotinib in patients with MET exon 14-altered advanced non-small cell lung cancer. $J$ Thorac Oncol. 2018;13 (10):S348. doi:10.1016/j.jtho.2018.08.300

63. Dong HJ, Li P, Wu CL, Zhou XY, Lu HJ, Zhou T. Response and acquired resistance to crizotinib in Chinese patients with lung adenocarcinomas harboring MET exon 14 splicing alternations. Lung Cancer. 2016;102:118-121. doi:10.1016/j.lungcan.2016.11.006

64. Heist RS, Sequist LV, Borger D, et al. Acquired resistance to crizotinib in NSCLC with MET exon 14 skipping. $J$ Thorac Oncol. 2016;11(8):1242-1245. doi:10.1016/j.jtho.2016.06.013

65. Schrock AB, Lai A, Ali SM, Miller VA, Raez LE. Mutation of MET Y1230 as an acquired mechanism of crizotinib resistance in NSCLC with MET exon 14 skipping. J Thorac Oncol. 2017;12(7): e89-e90. doi:10.1016/j.jtho.2017.02.017

66. Bahcall M, Sim T, Paweletz CP, et al. Acquired METD1228V mutation and resistance to MET inhibition in lung cancer. Cancer Discov. 2016;6 (12):1334-1341. doi:10.1158/2159-8290.CD-16-0686

67. Wolf J, Seto T, Han J-Y, et al. Capmatinib (INC280) in MET $\Delta$ ex14-mutated advanced non-small cell lung cancer (NSCLC): efficacy data from the phase II GEOMETRY mono-1 study. Journal of Clinical Oncology. 2019;37(15_suppl):9004-9004.

68. Felip E, Sakai H, Patel J, et al. OA12.01 phase II data for the MET inhibitor tepotinib in patients with advanced NSCLC and MET exon 14-skipping mutations. $J$ Thorac Oncol. 2018;13(10):S347. doi:10.1016/j.jtho.2018.08.299

69. Preliminary efficacy and safety results of savolitinib treating patients with pulmonary sarcomatoid carcinoma (PSC) and other types of non-small cell lung cancer (NSCLC) harboring MET exon 14 skipping mutations. Available from: http://cancerres.aacrjournals.org/content/79/ 13_Supplement/CT031. Published 2019. Accessed July 14, 2019.

70. Wu Y, Zhou J, Lu S et al. Educatioanal portal for oncologists. Phase II study of tepotinib + gefitinib (TEP+GEF) in MET-positive $(\mathrm{MET}+) /$ epidermal growth factor receptor (EGFR)-mutant (MT) non-small cell lung cancer (NSCLC). OncologyPRO. Available from: https://oncologypro. esmo.org/Meeting-Resources/ESMO-2018-Congress/Phase-2-study-of -tepotinib-gefitinib-TEP-GEF-in-MET-positive-MET-epidermal-gro wth-factor-receptor-EGFR-mutant-MT-non-small-cell-lung-cancer-NS CLC. Published 2018. Accessed April 15, $2019 .$.

71. Adding the MET inhibitor savolitinib to osimertinib was beneficial for certain pretreated lung cancer patients in TATTON trial. Available from: https://www.aacr.org/Newsroom/Pages/News-Release-Detail.aspx? ItemID=1294. Published 2019. Accessed July 14, 2019.

72. Roberts PJ, Der CJ. Targeting the Raf-MEK-ERK mitogen-activated protein kinase cascade for the treatment of cancer. Oncogene. 2007;26 (22):3291-3310. doi:10.1038/sj.onc. 1210422

73. Leonetti A, Facchinetti F, Rossi G, et al. BRAF in non-small cell lung cancer (NSCLC): pickaxing another brick in the wall. Cancer Treat Rev. 2018;66:82-94. doi:10.1016/j.ctrv.2018.04.006

74. Tissot C, Couraud S, Tanguy R, Bringuier PP, Girard N, Souquet PJ. Clinical characteristics and outcome of patients with lung cancer harboring BRAF mutations. Lung Cancer. 2016;91:23-28. doi:10.1016/j.lungcan.2015.11.006
75. Khunger A, Khunger M, Velcheti V. Dabrafenib in combination with trametinib in the treatment of patients with BRAF V600-positive advanced or metastatic non-small cell lung cancer: clinical evidence and experience. Ther Adv Respir Dis. 2018;12:1753466618767611. doi:10.1177/1753466618767611

76. Gautschi O, Milia J, Cabarrou B, et al. Targeted therapy for patients with BRAF-mutant lung cancer: results from the European EURAF cohort. J Thorac Oncol. 2015;10(10):1451-1457. doi:10.1097/ JTO. 0000000000000625

77. Planchard D, Kim TM, Mazieres J, et al. Dabrafenib in patients with $\mathrm{BRAF}(\mathrm{V} 600 \mathrm{E})$-positive advanced non-small-cell lung cancer: a single-arm, multicentre, open-label, phase 2 trial. Lancet Oncol. 2016;17(5):642-650. doi:10.1016/S1470-2045(16)00077-2

78. Planchard D, Smit EF, Groen HJM, et al. Dabrafenib plus trametinib in patients with previously untreated BRAF(V600E)-mutant metastatic non-small-cell lung cancer: an open-label, phase 2 trial. Lancet Oncol. 2017;18(10):1307-1316. doi:10.1016/S1470-2045(17)30679-4

79. Planchard D, Besse B, Kim TM, et al. Updated survival of patients (pts) with previously treated BRAF V600E-mutant advanced nonsmall cell lung cancer (NSCLC) who received dabrafenib (D) or $\mathrm{D}+$ trametinib (T) in the phase II BRF113928 study. J Clin Oncol. 2017;35(15_suppl):9075. doi:10.1200/JCO.2017.35.15_suppl.9075

80. U. S. FOOD \& DRUG ADMINISTRATION. FDA grants regular approval to dabrafenib and trametinib combination for metastatic NSCLC with BRAF V600E mutation. Updated June 22, 2017. Available from: https://www.fda.gov/Drugs/InformationOnDrugs/ ApprovedDrugs/ucm564331.htm. Published 2017. Accessed April 4, 2019.

81. Kim TW, Lee J, Shin SJ, et al. Belvarafenib, a novel pan-RAF inhibitor, in solid tumor patients harboring BRAF, KRAS, or NRAS mutations: Phase I study. Journal of Clinical Oncology. 2019;37(15_suppl):3000-3000.

82. Chan XY, Singh A, Osman N, Piva TJ. Role played by signalling pathways in overcoming BRAF inhibitor resistance in melanoma. Int J Mol Sci. 2017;18(7):1527. doi:10.3390/ijms 18071527

83. Nakagawara A. Trk receptor tyrosine kinases: a bridge between cancer and neural development. Cancer Lett. 2001;169(2):107-114. doi:10.1016/S0304-3835(01)00530-4

84. Asakura K, Ueda A, Kawamura N, Ueda M, Mihara T, Mutoh T. Clioquinol inhibits NGF-induced Trk autophosphorylation and neurite outgrowth in PC12 cells. Brain Res. 2009;1301:110-115. doi:10.1016/j.brainres.2009.09.011

85. Kaplan DR, Stephens RM. Neurotrophin signal transduction by the Trk receptor. J Neurobiol. 1994;25(11):1404-1417. doi:10.1002/ (ISSN) $1097-4695$

86. Stephens RM, Loeb DM, Copeland TD, Pawson T, Greene LA, Kaplan DR. Trk receptors use redundant signal transduction pathways involving SHC and PLC-gamma 1 to mediate NGF responses. Neuron. 1994;12(3):691-705. doi:10.1016/0896-6273(94)90223-2

87. Vaishnavi A, Le AT, Doebele RC. TRKing down an old oncogene in a new era of targeted therapy. Cancer Discov. 2015;5(1):25-34. doi:10.1158/2159-8290.CD-14-0765

88. Vaishnavi A, Capelletti M, Le AT, et al. Oncogenic and drug-sensitive NTRK1 rearrangements in lung cancer. Nat Med. 2013;19(11):1469-1472. doi:10.1038/nm.3352

89. Wang W, Xu C, Zhu Y, et al. P2.03-09 the real world of NTRK fusion data in the chinese lung cancer populations: a multicenter study. J Thorac Oncol. 2018;13(10):S719.

90. Kheder ES, Hong DS. Emerging targeted therapy for tumors with NTRK fusion proteins. Clin Cancer Res. 2018;24(23):5807-5814. doi:10.1158/1078-0432.CCR-18-1156

91. Berger S, Martens UM, Bochum S. Larotrectinib (LOXO-101). Recent Results Cancer Res. 2018;211:141-151.

92. Drilon A, Laetsch TW, Kummar S, et al. Efficacy of larotrectinib in TRK fusion-positive cancers in adults and children. $N$ Engl $J$ Med. 2018;378(8):731-739. doi:10.1056/NEJMoa1714448 
93. Farago A, Kummar S, Ibabekci S, et al. P1.13-40 rapid, robust and durable responses to larotrectinib in patients with TRK fusion non-small cell lung cancer. J Thorac Oncol. 2018;13(10):S597S598. doi:10.1016/j.jtho.2018.08.897

94. U. S. FOOD \& DRUG ADMINISTRATION. FDA approves larotrectinib for solid tumors with NTRK gene fusions. U. S. FOOD \& DRUG ADMINISTRATION. updated December 17, 2018 Available from: https://www.fda.gov/Drugs/InformationOnDrugs/ApprovedDrugs/ ucm626720.htm. Published 2018. Accessed April 4, 2019.

95. Drilon A, Nagasubramanian R, Blake JF, et al. A next-generation TRK kinase inhibitor overcomes acquired resistance to prior TRK kinase inhibition in patients with TRK fusion-positive solid tumors. Cancer Discov. 2017;7(9):963-972. doi:10.1158/2159-8290.CD17-0507

96. Demetri GD, Sigal D, Bazhenova L, et al. LBA17Efficacy and safety of entrectinib in patients with NTRK fusion-positive (NTRK-fp) tumors: pooled analysis of STARTRK-2, STARTRK-1 and ALKA-372-001. Ann Oncol. 2018;29(suppl_8). doi:10.1093/ annonc/mdx 807

97. Hervent AS, De Keulenaer GW. Molecular mechanisms of cardiotoxicity induced by ErbB receptor inhibitor cancer therapeutics. Int $\mathrm{J} \mathrm{Mol}$ Sci. 2012;13(12):12268-12286. doi:10.3390/ijms131012268

98. Takezawa K, Pirazzoli V, Arcila ME, et al. HER2 amplification: a potential mechanism of acquired resistance to EGFR inhibition in EGFR-mutant lung cancers that lack the second-site EGFRT790M mutation. Cancer Discov. 2012;2(10):922-933. doi:10.1158/21598290.CD-12-0108

99. Liu L, Shao X, Gao W, et al. The role of human epidermal growth factor receptor 2 as a prognostic factor in lung cancer: a meta-analysis of published data. J Thorac Oncol. 2010;5 (12):1922-1932. doi:10.1097/JTO.0b013e3181f26266

100. Albagoush SA, Limaiem F. HER2. [Updated 2018 Dec 29]. In: StatPearls. Treasure Island (FL): StatPearls Publishing; 2019 Jan-. Available from: https:/www.ncbi.nlm.nih.gov/books/NBK537134/.

101. Mazières $\mathrm{J}$, Barlesi F, Filleron T, et al. Lung cancer patients with HER2 mutations treated with chemotherapy and HER2-targeted drugs: results from the European EUHER2 cohort. Ann Oncol. 2016;27(2):281-286. doi:10.1093/annonc/mdv573

102. Lai WV, Lebas L, Barnes TA, et al. Afatinib in patients with metastatic or recurrent HER2-mutant lung cancers: a retrospective international multicentre study. Eur J Cancer. 2019;109:28-35. doi:10.1016/j.ejca.2018.11.030

103. Peters S, Curioni-Fontecedro A, Nechushtan H, et al. Activity of afatinib in heavily pretreated patients with ERBB2 mutation-positive advanced NSCLC: findings from a global named patient use program. $J$ Thorac Oncol. 2018;13(12):1897-1905. doi:10.1016/j.jtho.2018.07.093

104. Li BT, Shen R, Buonocore D, et al. Ado-trastuzumab emtansine for patients with HER2-mutant lung cancers: results from a phase II basket trial. J Clin Oncol. 2018;36(24):2532-2537. doi:10.1200/ JCO.2018.77.9777

105. Robichaux JP, Elamin YY, Tan Z, et al. Mechanisms and clinical activity of an EGFR and HER2 exon 20-selective kinase inhibitor in non-small cell lung cancer. Nat Med. 2018;24(5):638-646. doi:10.1038/s41591-018-0007-9

106. Heymach J, Negrao M, Robichaux J, et al. OA02.06 A phase II trial of poziotinib in EGFR and HER2 exon 20 mutant Non-Small Cell Lung Cancer (NSCLC). J Thorac Oncol. 2018;13(10):S323-S324. doi:10.1016/j.jtho.2018.08.243

107. Tsurutani J, Park H, Doi T, et al. OA02.07 updated results of phase 1 study of DS-8201a in HER2-expressing or -mutated advanced non-small-cell lung cancer. J Thorac Oncol. 2018;13(10):S324. doi:10.1016/j.jtho.2018.08.244

108. Chuang JC, Stehr H, Liang Y, et al. ERBB2-mutated metastatic non-small cell lung cancer: response and resistance to targeted therapies. J Thorac Oncol. 2017;12(5):833-842. doi:10.1016/j. jtho.2017.01.023
109. Riess JW, Gandara DR, Frampton GM, et al. Diverse EGFR exon 20 insertions and co-occurring molecular alterations identified by comprehensive genomic profiling of NSCLC. J Thorac Oncol. 2018;13(10):1560-1568. doi:10.1016/j.jtho.2018.06.019

110. Oxnard GR, Lo PC, Nishino M, et al. Natural history and molecular characteristics of lung cancers harboring EGFR exon 20 insertions. J Thorac Oncol. 2013;8(2):179-184. doi:10.1097/ JTO.0b013e3182779d18

111. Kobayashi Y, Mitsudomi T. Not all epidermal growth factor receptor mutations in lung cancer are created equal: perspectives for individualized treatment strategy. Cancer Sci. 2016;107 (9):1179-1186. doi:10.1111/cas.12996

112. Van Veggel B, Van Der Wekken A, Hashemi S, et al. P2.13-42 osimertinib treatment for patients with EGFR exon 20 insertion positive non-small-cell lung cancer. J Thorac Oncol. 2018;13(10): S815. doi:10.1016/j.jtho.2018.08.1437

113. Vyse S, Huang PH. Targeting EGFR exon 20 insertion mutations in non-small cell lung cancer. Signal Transduct Target Ther. 2019;4:5. doi:10.1038/s41392-019-0038-9

114. Spectrum pharmaceuticals provides poziotinib update. Available from: https://www.sppirx.com/release.html?id=21686. Published 2018. Accessed April 4, 2019.

115. A phase I/II study of TAK-788, an investigational EGFR/HER2 TKI, in previously treated advanced NSCLC with EGFR exon 20 insertion. Available from: https:/www.clinicaloptions.com/oncology/conferencecoverage/clin-onc-june-2019/lung-cancer/capsule-summary-slidesets /9007. Published 2019. Accessed July 18, 2019.

116. Muscarella LA, Rossi A. NRG1: a cinderella fusion in lung cancer? Lung Cancer Manag. 2017;6(4):121-123. doi:10.2217/1mt-20170018

117. Jonna S, Feldman RA, Swensen J, et al. Detection of NRG1 gene fusions in solid tumors. Clin Cancer Res. 2019;25(16):4966. doi:10.1158/1078-0432.CCR-19-0160

118. Yarden Y, Pines G. The ERBB network: at last, cancer therapy meets systems biology. Nat Rev Cancer. 2012;12(8):553-563. doi:10.1038/nrc3309

119. Jones MR, Lim H, Shen Y, et al. Successful targeting of the NRG1 pathway indicates novel treatment strategy for metastatic cancer. Ann Oncol. 2017;28(12):3092-3097. doi:10.1093/annonc/ $\operatorname{mdx} 523$

120. Drilon A, Somwar R, Mangatt BP, et al. Response to ERBB3-directed targeted therapy in NRG1-rearranged cancers. Cancer Discov. 2018;8(6):686-695. doi:10.1158/2159-8290.CD17-1004

121. Duruisseaux M, Liu SV, Han JY, et al. NRG1 fusion-positive lung cancers: clinicopathologic profile and treatment outcomes from a global multicenter registry. J Clin Oncol. 2019:9081.

122. Gay ND, Wang Y, Beadling C, et al. Durable response to afatinib in lung adenocarcinoma harboring NRG1 gene fusions. J Thorac Oncol. 2017;12(8):e107-e110. doi:10.1016/j.jtho.2017.04.025

123. Duruisseaux M, Laskin JJ, Tolba K, et al. Targeting NRG1-fusions in lung adenocarcinoma: afatinib as a novel potential treatment strategy. In: 2019 International Association for the Study of Lung Cancer World Conference on Lung Cancer; September 7-10, 2019; Barcelona, Spain. Abstract P114-25.

124. Turner N, Grose R. Fibroblast growth factor signalling: from development to cancer. Nat Rev Cancer. 2010;10(2):116-129. doi:10.1038/ $\operatorname{nrc} 2780$

125. Desai A, Adjei AA. FGFR signaling as a target for lung cancer therapy. J Thorac Oncol. 2016;11(1):9-20. doi:10.1016/j.jtho.2015.08.003

126. Cihoric N, Savic S, Schneider S, et al. Prognostic role of FGFR1 amplification in early-stage non-small cell lung cancer. $\mathrm{Br}$ J Cancer. 2014;110(12):2914-2922. doi:10.1038/bjc.2014.229

127. Dutt A, Ramos AH, Hammerman PS, et al. Inhibitor-sensitive FGFR1 amplification in human non-small cell lung cancer. PLoS One. 2011;6(6):e20351. doi:10.1371/journal.pone.0020351 
128. Fischer H, Taylor N, Allerstorfer S, et al. Fibroblast growth factor receptor-mediated signals contribute to the malignant phenotype of non-small cell lung cancer cells: therapeutic implications and synergism with epidermal growth factor receptor inhibition. Mol Cancer Ther. 2008;7(10):3408-3419. doi:10.1158/1535-7163. MCT-08-0444

129. Weiss J, Sos ML, Seidel D, et al. Frequent and focal FGFR1 amplification associates with therapeutically tractable FGFR1 dependency in squamous cell lung cancer. Sci Transl Med. 2010;2 (62):62ra93. doi:10.1126/scitranslmed.3001451

130. Li JJ, Yan S, Pan Y, et al. FGFR genes mutation is an independent prognostic factor and associated with lymph node metastasis in squamous non-small cell lung cancer. Cancer Biol Ther. 2018;19 (12):1108-1116. doi:10.1080/15384047.2018.1480294

131. Hashemi-Sadraei N, Hanna N. Targeting FGFR in squamous cell carcinoma of the lung. Target Oncol. 2017;12(6):741-755. doi:10.1007/s11523-017-0513-6

132. Rijavec E, Genova C, Barletta $\mathrm{G}$, et al. Investigational drugs targeting fibroblast growth factor receptor in the treatment of non-small cell lung cancer. Expert Opin Investig Drugs. 2017;26 (5):551-561. doi:10.1080/13543784.2017.1316714

133. Nintedanib in Treating Patients With Advanced Non-Small Cell Lung Cancer Who Have Failed Up to Two Previous Chemotherapy Regimens. Available from: https://clinicaltrials. gov/ct2/show/NCT01948141. Accessed April 4, 2019.

134. Nogova L, Sequist LV, Perez Garcia JM, et al. Evaluation of BGJ398, a fibroblast growth factor receptor 1-3 kinase inhibitor, in patients with advanced solid tumors harboring genetic alterations in fibroblast growth factor receptors: results of a global phase I, dose-escalation and dose-expansion study. J Clin Oncol. 2017;35 (2):157-165. doi:10.1200/JCO.2016.67.2048

135. BALVERSA ${ }^{\mathrm{TM}}$ (erdafitinib) receives U.S. FDA approval for the treatment of patients with locally advanced or metastatic urothelial carcinoma with certain FGFR genetic alterations. Available from: https://www.jnj.com/balversa-erdafitinib-receives-u-s-fda-approvalfor-the-treatment-of-patients-with-locally-advanced-or-metastatic-u rothelial-carcinoma-with-certain-fgfr-genetic-alterations. Published 2019. Accessed July 14, 2019.

136. Ji M, Guan H, Gao C, Shi B, Hou P. Highly frequent promoter methylation and PIK3CA amplification in non-small cell lung cancer (NSCLC). BMC Cancer. 2011;11:147. doi:10.1186/1471-2407$11-147$

137. Courtney KD, Corcoran RB, Engelman JA. The PI3K pathway as drug target in human cancer. J Clin Oncol. 2010;28(6):1075-1083. doi:10.1200/JCO.2009.25.3641

138. Dienstmann R, Rodon J, Serra V, Tabernero J. Picking the point of inhibition: a comparative review of $\mathrm{PI} 3 \mathrm{~K} / \mathrm{AKT} / \mathrm{mTOR}$ pathway inhibitors. Mol Cancer Ther. 2014;13(5):1021-1031. doi:10.1158/ 1535-7163.MCT-13-0639

139. Kawano O, Sasaki H, Endo K, et al. PIK3CA mutation status in Japanese lung cancer patients. Lung Cancer. 2006;54(2):209-215. doi:10.1016/j.lungcan.2006.07.006
140. Yamamoto H, Shigematsu H, Nomura M, et al. PIK3CA mutations and copy number gains in human lung cancers. Cancer Res. 2008;68(17):6913-6921. doi:10.1158/0008-5472.CAN-07-5084

141. Spoerke JM, O'Brien C, Huw L, et al. Phosphoinositide 3-kinase (PI3K) pathway alterations are associated with histologic subtypes and are predictive of sensitivity to PI3K inhibitors in lung cancer preclinical models. Clin Cancer Res. 2012;18(24):6771-6783. doi:10.1158/1078-0432.CCR-12-2347

142. Zhang L, Shi L, Zhao X, Wang Y, Yue W. PIK3CA gene mutation associated with poor prognosis of lung adenocarcinoma. Onco Targets Ther. 2013;6:497-502. doi:10.2147/OTT.S41643

143. Chaft JE, Arcila ME, Paik PK, et al. Coexistence of PIK3CA and other oncogene mutations in lung adenocarcinoma-rationale for comprehensive mutation profiling. Mol Cancer Ther. 2012;11 (2):485-491. doi:10.1158/1535-7163.MCT-11-0692

144. Sequist LV, Waltman BA, Dias-Santagata D, et al. Genotypic and histological evolution of lung cancers acquiring resistance to EGFR inhibitors. Sci Transl Med. 2011;3(75):75ra26. doi:10.1126/ scitranslmed.3002003

145. Bendell JC, Rodon J, Burris HA, et al. Phase I, dose-escalation study of BKM120, an oral pan-class I PI3K inhibitor, in patients with advanced solid tumors. J Clin Oncol. 2012;30(3):282-290. doi:10.1200/JCO.2011.36.1360

146. Soulieres D, Faivre S, Mesia R, et al. Buparlisib and paclitaxel in patients with platinum-pretreated recurrent or metastatic squamous cell carcinoma of the head and neck (BERIL-1): a randomised, double-blind, placebo-controlled phase 2 trial. Lancet Oncol. 2017;18(3):323-335. doi:10.1016/S1470-2045(17)30064-5

147. Hammerman PS, Sos ML, Ramos AH, et al. Mutations in the DDR2 kinase gene identify a novel therapeutic target in squamous cell lung cancer. Cancer Discov. 2011;1(1):78-89. doi:10.1158/ 2159-8274.CD-11-0005

148. Miao L, Wang $\mathrm{Y}, \mathrm{Zhu} \mathrm{S}$, et al. Identification of novel driver mutations of the discoidin domain receptor 2 (DDR2) gene in squamous cell lung cancer of Chinese patients. BMC Cancer. 2014;14:369. doi:10.1186/1471-2407-14-369

149. Haura EB, Tanvetyanon T, Chiappori A, et al. Phase I/II study of the Src inhibitor dasatinib in combination with erlotinib in advanced non-small-cell lung cancer. J Clin Oncol. 2010;28 (8):1387-1394. doi:10.1200/JCO.2009.25.4029

150. Johnson FM, Bekele BN, Feng L, et al. Phase II study of dasatinib in patients with advanced non-small-cell lung cancer. J Clin Oncol. 2010;28(30):4609-4615. doi:10.1200/JCO.2010.30.5474

151. Kelley MJ, Jha G, Shoemaker D, et al. Phase II study of dasatinib in previously treated patients with advanced non-small cell lung cancer. Cancer Invest. 2017;35(1):32-35. doi: $10.1080 / 07357907.2016 .1253710$

152. Trial of dasatinib in patients with advanced cancers harboring DDR2 mutation or Inactivating B-RAF mutation. Available from: https://clinicaltrials.gov/ct2/show/NCT01514864. Accessed April 8,2019 .
OncoTargets and Therapy

\section{Publish your work in this journal}

OncoTargets and Therapy is an international, peer-reviewed, open access journal focusing on the pathological basis of all cancers, potential targets for therapy and treatment protocols employed to improve the management of cancer patients. The journal also focuses on the impact of management programs and new therapeutic agents and protocols on patient perspectives such as quality of life, adherence and satisfaction. The manuscript management system is completely online and includes a very quick and fair peer-review system, which is all easy to use. Visit http://www.dovepress.com/ testimonials.php to read real quotes from published authors. 